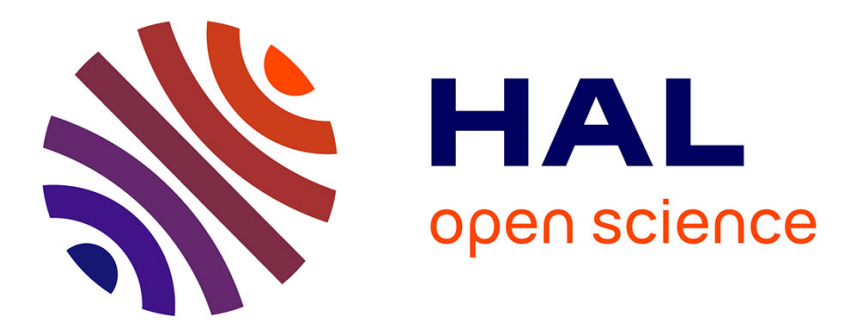

\title{
Lyl-1 marks and regulates primitive macrophages and microglia development
}

Shoutang Wang, Deshan Ren, Anna-Lila Kaushik, Gabriel Matherat, Yann

Lécluse, Dominik Filipp, William Vainchenker, Hana Raslova, Isabelle Plo, Isabelle Godin

\section{To cite this version:}

Shoutang Wang, Deshan Ren, Anna-Lila Kaushik, Gabriel Matherat, Yann Lécluse, et al.. Lyl-1 marks and regulates primitive macrophages and microglia development. 2020. hal-02992875

\section{HAL Id: hal-02992875 \\ https://hal.science/hal-02992875}

Preprint submitted on 6 Nov 2020

HAL is a multi-disciplinary open access archive for the deposit and dissemination of scientific research documents, whether they are published or not. The documents may come from teaching and research institutions in France or abroad, or from public or private research centers.
L'archive ouverte pluridisciplinaire HAL, est destinée au dépôt et à la diffusion de documents scientifiques de niveau recherche, publiés ou non, émanant des établissements d'enseignement et de recherche français ou étrangers, des laboratoires publics ou privés. 


\section{Lyl-1 marks and regulates primitive macrophages and microglia development}

Shoutang Wang ${ }^{1,{ }^{+}, *}$, Deshan $\operatorname{Ren}^{1,{ }^{++}, *}$, Anna-Lila Kaushik ${ }^{1,{ }^{++}}$, Gabriel Matherat ${ }^{1,++\dagger}$, Yann Lécluse $^{2}$, Dominik Filipp ${ }^{3}$, William Vainchenker ${ }^{1}$, Hana Raslova ${ }^{1}$, Isabelle Plo $^{1}$, Isabelle Godin ${ }^{1}$

${ }^{1}$ Gustave Roussy, INSERM U1287, Villejuif; Université Paris-Saclay, France.

2 PFIC, IUMS AMMICa (US 23 INSERM / UMS 3655 CNRS; Gustave Roussy, Villejuif, France

${ }^{3}$ Laboratory of Immunobiology, Institute of Molecular Genetics of the Czech Academy of Sciences, Prague, Czech Republic.

Present addresses: ${ }^{\dagger}$ Department of Pathology and Immunology, Washington University School of Medicine, St. Louis, MO, 63110, USA; ${ }^{++}$Medical school of Nanjing university, Model Animal Research Center, Nanjing University, Nanjing 210093, China; ${ }^{+\dagger \dagger}$ Plasseraud IP, 33064 Bordeaux, France; ${ }^{+++\dagger}$ INSERM U1016, CNRS UMR8104, Institut Cochin, Université de Paris, Paris, France * Equal contribution;

Short Title: Lyl-1 in macrophage/microglia ontogeny

\section{Corresponding author:}

Isabelle Godin (ORCID: 0000-0001-8577-8388)

INSERM U1287; Institut Gustave Roussy-PR1; 114, rue Edouard Vaillant; 94805 VILLEJUIF Cedex;

France

Email address: Isabelle.Godin@gustaveroussy.fr ;

Phone: (33) 1421141 43; Fax: (33) 142115240

\section{Key points:}

1- Lyl-1 expression marks yolk sac macrophages and brain macrophage/microglia/BAM.

2- Lyl-1 deficiency impairs primitive macrophage development and leads to the up-regulation of genes involved in embryo patterning.

3- Lyl-1-expressing primitive macrophages have an immuno-modulatory phenotype.

4- Lyl-1 deficiency impairs microglia development and the expression of genes involved in neurodevelopment. 


\section{Abstract}

During ontogeny, resident macrophages (MФs) of the nervous system emerge from haematopoietic stem cell-independent progenitors originating in the Yolk Sac (YS), so that factors impairing YS M $\Phi$ development may lead to neurodevelopmental disorders resulting from defective brain resident $M \Phi$.

Here we show that Lyl-1, a bHLH transcription factor related to Scl/Tal-1, marks primitive macrophage $\left(M \Phi^{\text {Prim }}\right)$ progenitors in the YS. Transcriptomic analysis of YS M $\Phi$ progenitors indicated that $M \Phi^{\text {Prim }}$ progenitors present at embryonic day (E) 9 are clearly distinct from those present at E10. Lyl-1 bHLH disruption led to an increased production and a defective differentiation of $\mathrm{M} \Phi^{\text {Prim }}$ progenitors. These differentiation defects were associated with profound modifications of the expression of genes involved in embryonic patterning and neurodevelopment. They also induced a reduced production of mature $M \Phi /$ microglia in the early brain, as well as a transient reduction of the microglia pool at midgestation and in the newborn.

We thus identify Lyl-1 as a critical regulator of $M \Phi^{\text {Prim }}$ and microglia development, which disruption may impair organogenesis, including neurodevelopment processes. 


\section{INTRODUCTION}

Amongst the components of the transcription factor network that regulate the various features of haematopoietic cells, Tal-1, Lmo2, Runx1 and Gata2 stand out as the major regulators of haematopoietic progenitor development during ontogeny (Pina and Enver, 2007; Wilson et al., 2010). Tal-1, Lmo2 and Gata-2 belong to a transcriptional complex, which also includes the basic helix-loop-helix (bHLH) transcription factor lymphoblastic leukaemia-derived sequence 1 (Lyl-1). Contrary to its paralog Tal-1, which is mandatory for the specification of all haematopoietic progenitors (Curtis et al., 2012; Porcher et al., 2017), the function of Lyl-1 during developmental haematopoiesis remains largely unknown. We therefore analysed this function using $L y \mid-1^{\text {Lacz/Lacz }}$ mutant mice (Capron et al., 2006), focusing on the initial steps of haematopoietic cells development in the yolk sac (YS).

During ontogeny, haematopoietic progenitors are generated in three successive waves (Palis, 2016). The two first occur in the YS prior to Haematopoietic Stem Cell (HSC) generation. This HSC-independent haematopoiesis comprises first the primitive haematopoietic wave, with the transient production of monopotent progenitors with embryonic specific features. The second YS wave, called transient-definitive, provides for a limited duration progenitors (mostly erythromyeloid) that seed the foetal liver (FL) and produce a haematopoietic progeny that displays definitive/adult differentiation features. Finally, HSC, generated in the aorta region in the third and definitive haematopoietic wave, immediately migrate to the FL where they mature and amplify to ultimately provide the population that will maintain lifelong haematopoiesis in the adult (Cumano and Godin, 2007; Kieusseian et al., 2012).

During YS haematopoiesis, $\mathrm{M} \Phi$ arising from the two waves derive either from a monopotent MФ progenitor in the primitive wave (Bertrand et al., 2005b; Palis et al., 1999) or from the progressive differentiation of EMP via the production of GM progenitors, and ultimately of 
granulocytes (G) and $M \Phi$ progenitors in the transient-definitive wave in a cMyb-dependent pathway (McGrath et al., 2015).

In the recent years, fate-mapping approaches aimed at determining the embryonic origin of tissue resident $M \Phi s$ indicated that most tissues harbour resident $M \Phi s$ of various origins, such as YS, FL and adult bone marrow (reviewed in (Ginhoux and Guilliams, 2016; Hoeffel and Ginhoux, 2018)), which complicates the characterisation of the functions of the various M $\Phi$ subsets. In the case of brain resident $\mathrm{M \Phi s,} \mathrm{fate-mapping} \mathrm{analyses} \mathrm{established} \mathrm{that,} \mathrm{contrary} \mathrm{to} \mathrm{others} \mathrm{tissue,}$

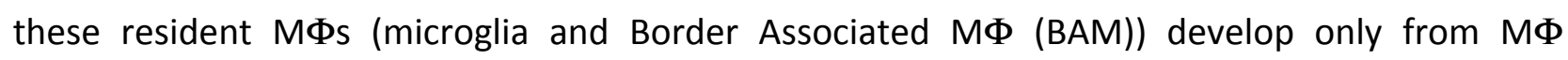
progenitors originating from the YS (Ginhoux et al., 2010; Gomez Perdiguero et al., 2015; Kierdorf et al., 2013; Schulz et al., 2012; Utz et al., 2020), confirming a developmental model we had previously put forward (Alliot et al., 1999). However, the wave (or waves) of origin remained unclear due to the limited criteria that discriminate progenitors from the two YS waves.

We here show that, at the early stages of YS haematopoiesis, $L y l-1$ expression discriminates primitive $\left(M \Phi^{\text {Prim }}\right)$ from transient-definitive $\left(M \Phi^{T-D e f}\right) M \Phi$ progenitors. In the brain, Lyl-1 marked the entire microglia/BAM population at the onset of brain colonisation and appeared to regulate microglia/BAM development.

Altogether, these data point to Lyl-1 as a major regulator of early embryonic $M \Phi$ progenitors development and advocate for further analyses to more precisely delineate Lyl-1 function during the development of resident $M \Phi$ in homeostatic and pathological contexts. 


\section{RESULTS}

\section{Lyl-1 expression discriminates $M \Phi^{\text {Prim }}$ from $M \Phi^{T-D e f}$ progenitors in the early YS}

Since $L y l-1$ is expressed in the YS from the onset of haematopoiesis (Giroux et al., 2007), we first explored its function by characterizing the progenitors produced by WT, $L y l-1^{W T / L a c Z}$ and $L y l-$ $1^{\text {LacZ/LacZ }}$ YS in clonogenic assay. To do so, YS at embryonic day (E) 8 were maintained in organ culture for 1 day (E8 OrgD1-YS), allowing only the development of progenitors from both primitive and transient-definitive waves (Cumano et al., 1996; Cumano et al., 2001). Compared to WT, the production of $M \Phi$ colonies was increased in $L y l-1^{W T / L a c Z}$ and $L y /-1^{L a c z / L a c z}$ OrgD1-YS. Otherwise, the clonogenic potential and colony distribution were unmodified (Fig. 1a).

Using FACS-Gal assay (Sup. fig 1a), we noticed that the entire $M \Phi$ progenitor population (cKit ${ }^{+} \mathrm{CD} 45^{+} \mathrm{CD} 11 \mathrm{~b}^{+}$) expressed Lyl-1 at E9. In contrast, from E9.5 (as in E8-OrgD1-YS), two MФ progenitor subsets discriminated by FDG/Lyl-1 expression were present (Fig. 1 b), suggesting that Lyl-1 may mark $M \Phi^{\text {Prim }}$ progenitors from the earliest wave. After E9.5, the YS harbours both $M \Phi^{\text {Prim }}$ and $M \Phi^{T-D e f}$ progenitors from the two YS waves and these two progenitors subsets cannot be discriminated by phenotype (Bertrand et al., 2005b). We therefore investigated the known features that discriminate the two YS waves such as the origin from monopotent progenitors beginning at E7.25 for primitive progenitors (Bertrand et al., 2005b; Palis et al., 1999) or, for the transient-definitive progenitors, their progressive differentiation from EMP via the production of GM progenitors, and ultimately of granulocytes $(\mathrm{G})$ and $\mathrm{M} \Phi$ progenitors (McGrath et $a l_{.}, 2015$ ) and the dependence on $c M y b$ expression for their production (Hoeffel et al., 2015;

\section{Schulz et al., 2012).}

FACS-Gal assay performed at E8 (0-5S), when only $M \Phi^{\text {Prim }}$ are present, demonstrated that all early $\mathrm{M} \Phi^{\text {Prim }}$ progenitors, characterised by a $\mathrm{CD} 11 \mathrm{~b}^{+} \mathrm{CD} 31^{+}$phenotype (Bertrand et al., 2005b), displayed FDG/Lyl-1 expression (Fig. 1C). Most FDG ${ }^{+} /$Lyl- $1^{+}$cells $\left(69.27 \% \pm 0.33 \%\right.$ ) from $L y /-1^{\text {WT/Lacz }}$ 
E8-YS co-expressed CD11b and CD31 and consistently produced $M \Phi$ colonies $(72.78 \pm 9.65 \% ; n=3)$ in clonogenic assays, amounting 1-4 MФ progenitors per YS, a value consistent with previously published data (Bertrand et al., 2005b; Palis et al., 1999).

As $M \Phi^{\mathrm{T}-\text { Def }}$ arise from EMPs that also generate GM progenitors and granulocytes (McGrath et al., 2015), we characterized the differentiation potential of $\mathrm{FDG}^{+} / \mathrm{Lyl}^{-} \mathrm{1}^{+}$and $\mathrm{FDG}{ }^{-} / \mathrm{Lyl}^{-} \mathbf{1}^{-}$fractions of myeloid progenitors (Ter119 ${ }^{-} \mathrm{KKit}^{+} \mathrm{CD} 45^{+} \mathrm{CD} 11 \mathrm{~b}^{+}$) from E10 YS in clonogenic assay (Fig. 1d). All samples produced few non-myeloid contaminants, such as EMk and EMP in similar, nonsignificant amounts. Similar to WT E9-YS M $\Phi^{\text {Prim }}$ progenitors, E10 $\mathrm{FDG}^{+} / \mathrm{Lyl}^{-} 1^{+}$progenitor subset nearly exclusively produced $M \Phi$ colonies, confirming the restriction of Lyl-1 expression to $M \Phi^{\text {Prim }}$ progenitors. In contrast, E10 FDG ${ }^{-} / \mathrm{Lyl}^{-} \mathbf{1}^{-}$myeloid progenitors produced $\mathrm{GM}, \mathrm{G}$ and $\mathrm{M} \Phi$ colonies, confirming their multipotent/transient-definitive status.

The restriction of Lyl-1 expression to $M \Phi^{\text {Prim }}$ progenitors was also strengthened by RT-qPCR comparison of $c M y b$ expression: $\mathrm{FDG}^{-} / \mathrm{Lyl}^{-1} \mathbf{1}^{-}$progenitors displayed $\mathrm{CMyb}$ levels similar to Lineage negative Sca $1^{+} \mathrm{CKit}^{+}$progenitors from E12-FL, whereas, as E9-YS $\mathrm{M} \Phi^{\text {Prim }}$ progenitors, $\mathrm{FDG}^{+} / \mathrm{Lyl}^{-} \mathrm{1}^{+}$ YS progenitors expressed low cMyb levels, strengthening their primitive status (Fig. 1e).

A distinct separation of E9 and E10 M $\Phi$ progenitors was also confirmed in RNA-seq analysis of $\mathrm{M} \Phi$ progenitors $\left(\mathrm{CD} 45^{+} \mathrm{CD} 11 \mathrm{~b}^{+} \mathrm{cKit}^{+}\right)$sorted at $\mathrm{E9}$, when the $\mathrm{YS}$ contains only $\mathrm{M} \Phi^{\text {Prim }}$ progenitors, and at E10, when it contains both $M \Phi^{\text {Prim }}$ and $M \Phi^{\text {T-Def }}$ progenitors. Principal Component Analysis pointed to a separation between E9 and E10 samples in both WT and Lyl-1 ${ }^{\text {LacZ/LacZ }}$ YS (PC1) and a separation between WT and $L y l-1^{\text {LacZ/LacZ }}$ samples (PC2) clearer at E9 in frame with the restriction to $\mathrm{M} \Phi^{\text {Prim }}$ progenitors at this stage (Fig. 1f).

E9 and E10 WT MФ progenitors differed by the expression of 726 genes. Amongst the differentially expressed genes (DEGs), 176 were up-regulated at E9 and 550 at E10. Considering the presence of both $M \Phi^{\text {Prim }}$ and $M \Phi^{T-D e f}$ progenitors in E10-YS, the DEGs found at this stage may 
reflect either waves-specific differences or stage-dependent changes related to the maturation of $\mathrm{M} \Phi^{\text {Prim }}$ progenitors.

Overlapping the identified DEGs to those obtained by Mass et al. (Mass et al., 2016) to characterise EMP and E10.25-E10.5 MФs confirmed that E9 $M \Phi^{\text {Prim }}$ progenitors were clearly distinct from these two populations. While about 5\% of E10 WT up-regulated genes belonged to the EMP and M $\Phi$ signatures, these genes represented respectively $27.5 \%$ and $35.6 \%$ of these signatures (Fig. 1g). A similar separation was also observed in Gene Set Enrichment Analysis (GSEA) (Sup. fig。1b). These observations suggest that within E10 M $\Phi$ progenitors some, likely the $M \Phi^{T-D e f}$ ones, retain part of the EMPs signature.

Focusing on the differences with E10 M $\Phi$ progenitors in a WT context, E9 M $\Phi^{\text {Prim }}$ progenitors were found to differ from E10 progenitors by their transcription factors (TF) repertoire. E10 WT $M \Phi$ progenitors were enriched in genes associated with erythroid development (Gata1, Gata2,

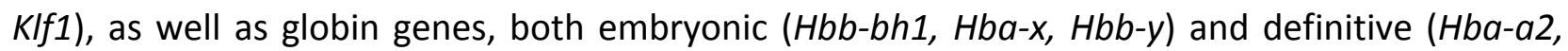
Hba-a1, Hbb-bt) (Fig. 1g). E9 and E10 MФ progenitors both exhibited a high expression of Spi1/PU.1 compared to Gata1 (Fig. 1h). The higher expression level of erythroid genes and of genes involved in granulo-monocytic (Mpo, Csf2r/GM-CSF receptors, Cebp, Jun) and megakaryocytic development (Pf4, TPO signalling) (Fig. 1g, Sup. table 1) at E10 sustains the notion that $M \Phi^{T-D e f}$ progenitors retain the expression of genes that characterize their EMP ancestor, as well as the monopotent status of E9 progenitors (Fig.1d).

Altogether, these data validate the monopotent/primitive status of E9 $M \Phi$ progenitors. However, due to the simultaneous presence of $M \Phi^{\text {Prim }}$ and $M \Phi^{T-D e f}$ progenitors in $E 10-Y S$, the differential expressions of makers that are wave-specific at this stage, such as $L y /-1$ for the primitive wave and cMyb and TIr2 (Balounova et al., 2019) for the transient-definitive one, were 
not significant despite a net tendency to a decrease for $L y l-1$ and an increase for $c M y b$ and $T / r 2$ (Sup. fig. 1c).

Through QIAGEN's Ingenuity ${ }^{\circledR}$ Pathway (IPA) and GSEA analyses, it appeared that $M \Phi^{\text {Prim }}$ progenitors were more active in Eicosanoid signalling than E10 progenitors (Fig. 1i). E9 $M \Phi^{\text {Prim }}$ progenitors were also enriched in type I interferon (IFN) $\beta$ and type II IFN $\gamma$ signalling (Fig. Ij) and, as a consequence, in MHC-II related genes, especially Cd74 (top 1 IPA network) (Fig. 1k; Sup. fig. 1d). Flow cytometry analyses confirmed a low, but significant, enrichment of MHC-II expression in E9 $\mathrm{M} \Phi$ compared to $\mathrm{E} 10$ progenitors (Sup. fig. 1e). For their part, E10 M $\Phi$ progenitors were more active in inflammatory signalling (Fig. 1i, l; Sup. fig. 1d, f-h; Sup. table 1). They were also more metabolically active than E9 M $\Phi$ progenitors (Sup. table 1). In addition, the complement cascade and phagocytosis also prevailed at E10 (Sup. fig. 1i, j).

\section{Lyl-1 deficiency in $M \Phi^{\text {Prim }}$ leads to patterning defects}

During YS development, both $M \Phi^{\text {Prim }}$ and $M \Phi^{\mathrm{T} \text {-Def }}$ progenitors originate from $\mathrm{CKit}^{+} \mathrm{CD} 31^{+} \mathrm{CD} 45^{-}$

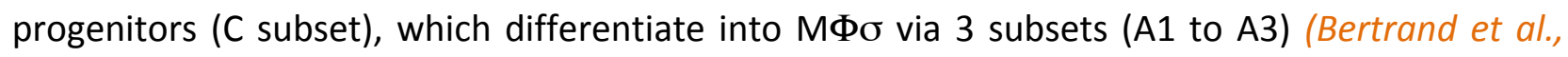
2005b) (Sup. fig. 2a). When evaluating the effect of Lyl-1 deficiency at the earliest stage of $M \Phi^{\text {Prim }}$ development, both FACS-Gal and clonogenic assays pointed to an increased production

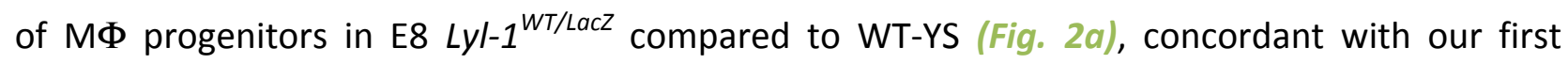
observation (Fig. 1a). The high increase of the expression level of Itga2b/CD41 in Lyl-1 ${ }^{\text {lacz/lacz }}$ $M \Phi^{\text {Prim }}$ progenitors (Fig. 2b) may reflect the elevated commitment of mesodermal/prehaematopoietic cells to a $\mathrm{M} \Phi$ fate (Sup. data and sup fig. 2b-d).

We noticed a clear-cut modification of the TF network (Fig. 2c) that controls developmental haematopoiesis (Wilson et al., 2010): beside the expected reduction of Lyl-1 expression, the expression of Lmo2, a target of Lyl-1 (McCormack et al., 2013), was also down-regulated, while 
Tal-1 up-regulation might reflect a compensatory role (Curtis et al., 2012). The consequence of these changes on early stages of YS development were apparent in GSEA analyses: both pathways and GO terms uncovered a general up-regulation of signalling pathways involved in

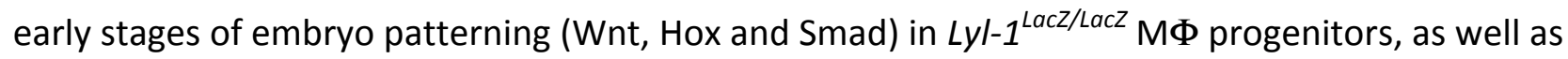
deep changes in collagen, integrin and cadherin usage (Sup. table 2). Accordingly, various developmental trajectories were affected (Fig. 2d), with an up-regulation in E9 Lyl-1 ${ }^{\text {Lacz/LacZ } M \Phi ~}$ progenitors of gene sets related to "anterior-posterior pattern specification" and "anatomical structure formation involved in morphogenesis", notably skeletal and nervous system development.

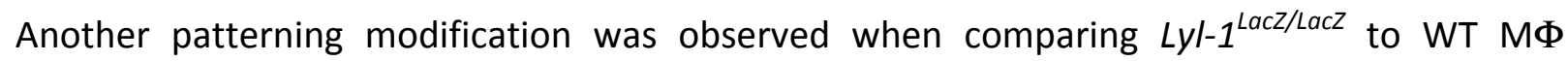
progenitors at E10: at this stage, GSEA and KEGG analyses pointed to the down-regulation of gene sets involved in heart development (Sup. fig. 2e; Sup. table 3). The heart harbours three resident $M \Phi$ subsets, two of which originate from the YS. Some of the features that differentiate the two CCR2 ${ }^{-}$YS-derived subsets (Epelman et al., 2014) also characterize $E 9 M \Phi^{\text {Prim }}$ progenitors (high MHC-II expression (Fig. 1k) and low phagocytosis ability (Sup. fig. 1j). Therefore, a function for $L y l-1$ in heart development may be considered.

These patterning defects, which appear triggered by defective $M \Phi^{\text {Prim }}$ may be responsible, at least in part, for the significant decreased of litter size and increased perinatal lethality (Sup. fig. 2f) observed in $L y l-1^{\text {Lacz/Lacz }}$ mice compared to WT, which indicates the requirement for functional Lyl-1 during various developmental processes.

\section{Defective $M \Phi^{\text {Prim }}$ development in Lyl-1 mutants}

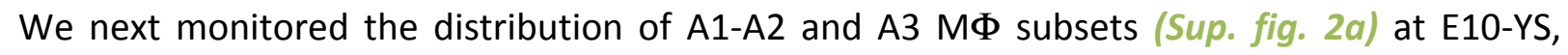
when all three subsets are present, using the $C \times 3 c r 1^{\text {WT/GFP }}:$ Lyl-1 ${ }^{\text {LacZ/LacZ }}$ double knock-in strain. The 
analysis of $L y l-1$ expression in A1-A2-A3 subsets from $C \times 3 C r 1^{W T / G F P}$ YS at E10 indicated that $L y /-1$ is expressed throughout $M \Phi$ differentiation, with a level decreasing from A1 to A3 (Sup. fig. 3a).

The subset distribution was highly impacted by $L y \mid-1$ deficiency, leading to an increased A1 and a reduced $A 2$ and $A 3$ pool sizes (Fig. 3a). Thus, Lyl-1 appears to regulate $M \Phi$ progenitors differentiation towards mature $M \Phi$. This defective differentiation could originate from the altered cytokine signalling uncovered in E9 mutant progenitors through GSEA and IPA analyses (Fig. 3b; Sup. fig. 3b). A limited or delayed differentiation of E9 $M \Phi^{\text {Prim }}$ progenitors was supported by the decreased expression of Ptprc/CD45, Csfr1, Itgam/CD11b and CD33 (Fig. 3c). The PU.1 signalling pathway was also decreased in E9 Lyl-1 ${ }^{\text {Lacz/LacZ }}$ progenitors (Sup. fig. 3c; Sup. table 4b).

Lyl-1 $1^{\text {LacZ/LacZ } M \Phi ~ p r o g e n i t o r s ~ w e r e ~ a l s o ~ d e f i c i e n t ~ i n ~ t h e ~ I F N ~ s i g n a l l i n g ~ t h a t ~ c h a r a c t e r i z e ~ E 9 ~}$ $\mathrm{M} \Phi^{\text {Prim }}$ progenitors, notably Irf8, a factor involved in the development of YS-M $\Phi$ and microglia (Hagemeyer et al., 2016; Kierdorf et al., 2013) (Fig. 3d). Compared to WT progenitors,

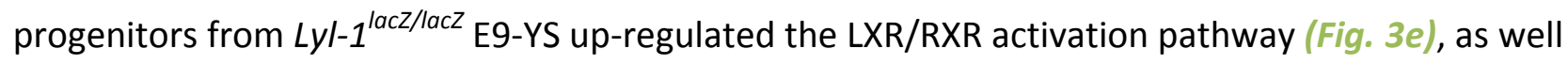
as various metabolic pathways, including some enriched in E10 WT progenitors (Butanoate and steroid) (Sup. table 1), and other which were not (Fructose/mannose and Fatty acid) (Fig. 2d). They were also less active in inflammatory signalling pathways, particularly through NFkB, a factor known to interact with Lyl-1 (Ferrier et al., 1999), and in TLR signalling (TIr4, TIr7, TIr8, TIr9) (Fig. 3d; Sup. fig. 3b, d-e; Sup. table 4b).

To better identify the core signature of $L y l-1$ deregulation taking into account the maturation

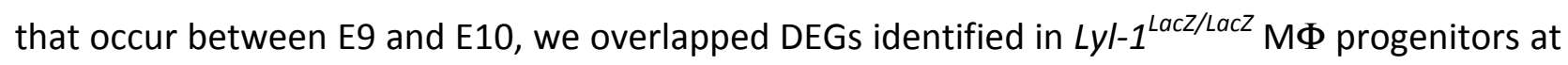
both stages. From the 16 DEGs common to both stages (Fig. 3f), only one, Fcgr2b, showed an opposite deregulation during development, being first down-regulated in the mutant at $\mathrm{E}$, then expressed at higher levels than in the WT at E10. 


\section{Lyl-1 ${ }^{+} M \Phi$ progenitors contribute to the early brain and foetal liver.}

The foetal liver (Kieusseian et al., 2012) and brain (Alliot et al., 1999; Ginhoux et al., 2010) are both colonised as early as E9 by YS-derived resident M $\Phi$ progenitors. We therefore implemented a FACS-Gal assay to evaluate the contribution of Lyl-1-expressing $M \Phi^{\text {Prim }}$ progenitors to these rudiments at E10 (Fig. 4a). While E10-YS comprised both $\mathrm{FDG}^{+} / \mathrm{Lyl}^{+} \mathrm{1}^{+}$and $\mathrm{FDG}^{-} / \mathrm{Lyl}^{-} \mathrm{1}^{-} \mathrm{M \Phi}$ progenitor and mature $\left(\mathrm{F} 4 / 80^{+}\right) \mathrm{M} \Phi$ subsets (Fig. 1b), the brain from the same embryos essentially harboured $\mathrm{FDG}^{+} / \mathrm{Lyl}-1^{+} \mathrm{M} \Phi$ progenitors and mature $\mathrm{M} \Phi$. In contrast, $\mathrm{M} \Phi$ progenitors

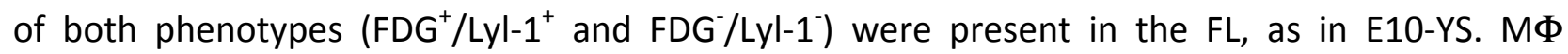
progenitors were more abundant in mutant FL than in WT (Fig. 4b), as in the YS, but the amount of mature $M \Phi$ was unmodified (Data not shown).

We next focused on brain $M \Phi$ during the colonisation stage, which occurs until E11 (Matcovitch-Natan et al., 2016). At this stage, microglia and perivascular, meningeal and choroid plexus $M \Phi$, collectively refered to as BAMs, are all located in the brain mesenchyme and are therefore undistinguishable (Goldmann et al., 2016; Utz et al., 2020). FACS-Gal assay demonstrated that the whole $\mathrm{F} 4 / 80^{+}$microglia/BAM population expresses Lyl-1 throughout the settlement period (Fig. 4c). The presence of $\mathrm{Lyl}-\mathrm{-}^{+} \mathrm{F} 4 / 80^{+}$microglia/BAM at the earliest stage of brain colonisation suggests that already differentiated $M \Phi$ could participate to the colonization step.

A lineage relationship between $\mathrm{Lyl}-1^{+} \mathrm{M} \Phi^{\text {Prim }}$ progenitors and early microglia/BAM is therefore strongly considered, based on the timing of their appearance and on similar features, such as the low level of $c M y b$ expression (Fig. 4d), concordant with the cMyb-independent development of microglia (Kierdorf et al., 2013; Schulz et al., 2012). Such a putative lineage relationship is also supported by our RNA-seq data. WT E9 $M \Phi^{\text {Prim }}$ progenitors expressed 
significantly lower levels of Mrc1/CD206 than E10 M $\Phi$ progenitors, as well as a significantly higher level of Sall3 and a slight increase of Sall1 (Fig. 4e), a transcriptomic pattern that characterises microglia (Lavin et al., 2014; Mass et al., 2016; Matcovitch-Natan et al., 2016). This pattern suggests that E9-YS M $\Phi^{\text {Prim }}$ progenitors show a partial bias toward a microglia signature. These observations point to $\mathrm{E} 9 \mathrm{M} \Phi^{\text {Prim }}$ progenitors as a likely source of embryonic microglia with the first stage of microglia development program already initiated in YS M $\Phi^{\text {Prim }}$ progenitors at E9.

We next examined the distribution of $A 1-A 2$ and $A 3 M \Phi$ subsets in the brain of $E 10$ Cx3Cr1 ${ }^{\text {WT/GFP }}:$ Lyl-1 ${ }^{\text {LacZ/LacZ }}$ embryos to assess the impact of Lyl-1 deficiency at the onset of brain colonisation. $L y /-1$ expression levels in the brain $M \Phi$ subsets were similar to those observed in the YS (Fig. 4f, Sup. fig. 3a). However, Lyl-1 deficiency led to an increased A1 and a reduced A3 pool size (Fig. 4g), indicating that in the brain, as in the YS, Lyl-1 regulates the differentiation of $M \Phi$ progenitors towards mature microglia/BAM.

\section{Lyl-1 inactivation impairs microglia development at two development stages}

Having defined Lyl-1 implication during microglia/BAM settlement in the brain, we turned to later development stages, focusing on $\mathrm{CD} 45^{\mathrm{lo}}$ microglia. Cytometry and database analyses (Matcovitch-Natan et al., 2016) confirmed the continuous expression of Lyl-1 in microglia until adulthood (Sup. fig. 4a). We therefore examined the impact of $L y l-1$ inactivation on microglia pool size during development. Microglia quantification pointed to E12 as the first step impacted by the mutation. The arrested increase of the microglia pool in $L y l-1^{\text {Lacz/LacZ }}$ brain at E12 (Fig. 5a) resulted from a reduced proliferation (Fig. 5b) rather than an increased apoptosis (Sup. fig. 4b). Moreover, Lyl-1 deficiency also provoked morphological changes in E12 Cx3cr1 ${ }^{\text {WT/GFP }}:$ Lyl-1 ${ }^{\text {LacZ/Lacz }}$ microglia, which displayed a reduced number and extent of ramifications compared to Cx3cr1 ${ }^{\text {WT/GFP }}$ microglia (Fig. 5c; Sup. fig. 4c, d). Interestingly, from E14, the microglia pool size 
returned to levels similar to WT embryos (Fig. 5a), a recovery that may result from the sharp reduction of apoptosis in Lyl-1 ${ }^{\text {Lacz/Lacz }}$ microglia at E14 (Sup. fig. 4b).

P0-P3 was identified as a second development stage altered in $L y /-1^{\text {LacZ/LacZ }}$ microglia. At birth, the cellularity of $L y l-1^{L a c Z / L a c Z}$ brain was significantly decreased compared to WT (Fig. $\left.5 d\right)$, which was not the case at earlier stages (Sup. fig. 4e). The recovery of CD11b ${ }^{+}$cells was also reduced (WT: $\left.140.96 \pm 0.91 \times 10^{3} ; n=9 ; L y /-1^{\text {LacZ/Lacz }}: 87.18 \pm 0.37 \times 10^{3} ; n=9\right)$. Consequently, Lyl-1 deficiency triggered a nearly 2 -fold reduction of the microglia population (Fig. $5 d$ ). This perinatal reduction of the microglia pool appeared transient, since no difference with WT brain was observed in the adult (Sup. fig. 4f). At perinatal stages, the reduction of brain cellularity in $L y /$ - $1^{\text {Lacz/Lacz }}$ mice points to Lyl-1 as a possible regulator of the trophic function of microglia on brain cells (Antony et al., 2011; Ueno et al., 2013).

The identification of E12 and P0-P3 as key stages for Lyl-1 function in microglia development was confirmed by RT-qPCR analyses of the expression of genes essential for M $\Phi$ (Spi1/PU.1, Csf1r, Mafb) and/or microglia (Runx1, Cx3cr1, Irf8) development and function, as well as known regulators of developmental haematopoiesis (Tal-1, Lmo2, Runx1) and related factors (Tcf3/E2A, Tcf4/E2.2) (Fig. 5e, f; Sup. fig. 4g). Time course analyses highlighted the down-regulation of Csf1r, Irf8 and Lmo2 in Lyl-1 ${ }^{\text {Lacz/LacZ }}$ microglia at both E12 and P0-P3, while Cx3cr1 was only decreased at E12 (Fig. 5g). Note that Lyl-1 expression was unmodified in Cx3Cr1 ${ }^{\text {GFP/GFP }}$ mutants (Fig. 5g). Interestingly, CX3cr1, as well as Irf8 and Lmo2, belong to potential Lyl-1 target genes (Wilson et al., 2010).

Mafb expression levels in $L y l-1^{L a c Z / L a c Z}$ microglia transiently decreased at PO-P3 and later returned back to WT expression levels (Fig. 5h). As Mafb represses resident M $\Phi$ self-renewal (Soucie et al., 2016), this transient decrease may be linked to the recovery of a normal amount of microglia after perinatal stages. Spi1/PU.1, Tcf3/E2A and Tcf4/E2.2 expression levels were 
unmodified in $L y /-1^{\text {Lacz/LacZ }}$ microglia at any stage, while Runx1 expression was only affected after birth. The expression of Tal-1 was decreased at E14 and increased after birth, suggesting that this Lyl-1 paralog (Curtis et al., 2012) does not compensate for Lyl-1 deficiency during embryonic stages of microglia development, but may do so during postnatal stages (Sup. fig. $4 \mathrm{~g}$ ).

Remarkably, our RNA-seq results indicate that the expression level of some of the genes deregulated in $L y /$ - $^{\text {LacZ/LacZ }}$ microglia at E12 and in the new-born were also down-regulated in $L y /$ -

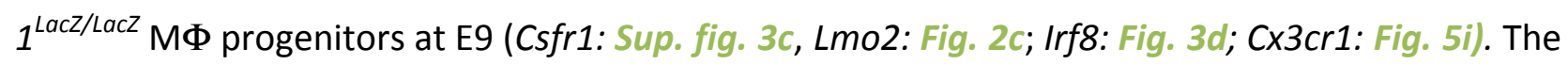
de-regulation of these genes in microglia was transient, however in both locations and stages

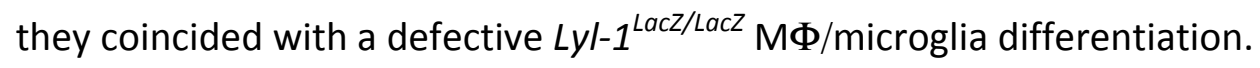

Other genes enriched in microglia (Fcrls, Mef2c, Maf) (Crotti and Ransohoff, 2016) or involved in the maintenance of microglia homeostasis (P2ry12) (Bisht et al., 2018) were also expressed in

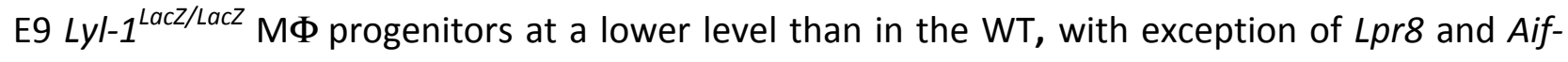
1/lba1 which expression levels were increased (Fig. 5i). The deregulation of these genes might underlie the relationship between $M \Phi^{\text {Prim }}$ progenitors and microglia/neural development which became apparent upon $L y /$-1 inactivation considering the large number of neural signalling pathways up-regulated in E9 $L y /-1^{\text {LacZ/LacZ }} \mathrm{M} \Phi$ progenitors (Fig. 2d) and the relationship of the DEGs enriched in E9 $M \Phi^{\text {Prim }}$ progenitors with brain formation and neuro-development uncovered in IPA analysis (Sup. fig. 4h).

Altogether, these data suggest that Lyl-1 deficiency in microglia may lead to neurodevelopmental defects. 


\section{DISCUSSION}

We here identified Lyl-1 as a marker for YS-derived $\mathrm{M} \Phi^{\text {Prim }}$ progenitors and found that, at the earliest stage of YS development, this expression discriminated $M \Phi^{\text {Prim }}$ progenitors from those produced in the transient-definitive waves. The early expression of Lyl-1 in YS mesoderm (Giroux et al., 2007), where it cannot substitute for the mandatory function of its paralog Tal-1 for the generation of haematopoietic progenitors (Porcher et al., 2017), has already been established (reviewed in (Curtis et al., 2012)). More recently, Lyl-1 was also identified as a regulator of mesoderm cell fate in human induced pluripotent stem cells (iPSC) (Palpant et al., 2017), and its function in the maintenance of primitive erythroid progenitors has been described (Chiu et al.,

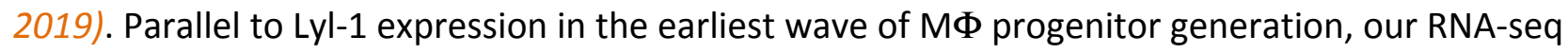
data now reveal Lyl-1 as an essential regulator in the specification of YS mesoderm to a haematopoietic fate since its invalidation affects major sets of genes involved in embryo patterning. Whether Lyl-1 role during the onset of organogenesis is specific to mesodermal stage or related to a patterning function for $M \Phi^{\text {Prim }}$ progenitors remains to be determined. Lyl-1 involvement in two discrete developmental steps of embryogenesis would constitute yet another similarity with its paralog Tal-1, which regulates both YS mesoderm determination to a haematopoietic fate and the differentiation of primitive erythroid cells (Curtis et al., 2012).

Within the $M \Phi$ lineage, Lyl-1 function during normal development would initially consist to restrict the size of the $M \Phi^{\text {Prim }}$ progenitor pool and/or the duration of its production, which is thought to be transient (McGrath et al., 2015), as shown by the increased size of this pool observed in Lyl-1 ${ }^{\text {LacZ/LacZ }}$ E8-E9 YS. Lyl-1 would also promote $M \Phi$ differentiation, when this process starts at $\mathrm{E9.5}$, as the maintenance of an increased size of $\mathrm{M} \Phi^{\text {Prim }}$ progenitor pool in $L y /$ $1^{\text {LacZ/LacZ }}$ E10-YS appears to result from a defective cytokine signalling. 
We also defined a signature for WT M $\Phi^{\text {Prim }}$ progenitors at E9, which comprises the Eicosanoid, MHC-II and IFN signalling pathways, pointing to an immuno-modulatory function for these $\mathrm{M} \Phi^{\text {Prim }}$ progenitors. Comparatively, $\mathrm{M} \Phi$ progenitors at $\mathrm{E} 10$ were rather involved in the response to bacteria and viruses, through phagocytosis and inflammatory signalling, with the preferential expression of TNF, TLR and TGF $\beta$ signalling pathways. Unfortunately, the simultaneous presence of $M \Phi^{\text {Prim }}$ and $M \Phi^{\text {T-Def }}$ progenitors at E10 makes it difficult to attribute the changes of gene expression to a stage dependent maturation of $M \Phi^{\text {Prim }}$ progenitors or to a signature specific to $M \Phi^{T-D e f}$ progenitors. However it indicates that most pathways favoured by $M \Phi$ progenitors at E10 were insensitive to $L y l-1$ invalidation, except TLR signalling pathway that was downregulated in E9 mutant $\mathrm{M} \Phi$ progenitors, compared to $\mathrm{WT}$.

The limitations of the current Lyl-1 model call for the development of new mouse models that will allow lineage specific reports of Lyl-1 expression and function in endothelial or haematopoietic lineages during discrete steps of early YS development and beyond. These extended investigations would probably improve our knowledge of other HSC-independent lineages, and benefit to the Embryonic Stem Cells/iPSC field in which the production of definitive cells types is complicated by the poor discrimination between primitive and definitive populations.

When analysing the contribution of $\operatorname{Lyl}-1^{+} \mathrm{M} \Phi$ progenitors to resident $M \Phi$, we detected their presence in $\mathrm{FL}$ and brain rudiments right from the onset of their colonisation. In the developing brain, microglia/BAM maintained Lyl-1 expression and shared with $M \Phi^{\text {Prim }}$ progenitors a low level of $c M y b$ expression. While fate-mapping analyses were instrumental to firmly ascribe a YS origin to microglia (Ginhoux et al., 2010) (Kierdorf et al., 2013; Schulz et al., 2012) (Gomez Perdiguero et al., 2015), this strategy could not ascertain which YS progenitor wave contributes to microglia (Mass, 2018; McGrath et al., 2015; Wittamer and Bertrand, 2020). Indeed, an 
independent/specific targeting of only one $M \Phi$ progenitors wave was prevented by: 1 - the similar phenotype of $\mathrm{M} \Phi^{\text {Prim }}$ and $\mathrm{M} \Phi^{\mathrm{T}-\mathrm{Def}}$ progenitors (Bertrand et al., 2005b); 2- the short timespan separating the two YS waves (E7.25 and E8.25); 3- the high variability of development stages of individual embryos within a litter at these stage (Downs and Davies, 1993) and 4- the duration of TAM induction, now admitted to last up to 72h (Senserrich et al., 2018). Using an ex vivo approaches whereby expression and potential can be examined in individually staged embryos, we found that, at least between E8 and E10, Lyl-1 expression discriminates $M \Phi^{\text {Prim }}$ from EMP-derived $M \Phi^{\mathrm{T} \text {-Def }}$ progenitors, and also marks the entire embryonic microglia/BAM populations.

We therefore favour a model ascribing microglia origin to Lyl-1 $M \Phi^{\text {Prim }}$ progenitors, as they preferentially expressed genes belonging to the microglia signature (Sall1 and Sall3, P2ry12, Mef2c, Fcrls), while E10 M $\Phi$ progenitors favoured the expression of genes (Id3, Runx3), belonging to resident $\mathrm{M} \Phi$ signatures in the lung and skin (Mass et al., 2016). Even though a later contribution of $M \Phi^{\text {T-Def }}$ progenitors could be considered, provided they up-regulate Lyl-1 expression at a later stage, such model is supported by the low level of $c M y b$ expressed by both Lyl-1 $1^{+} M \Phi^{\text {Prim }}$ progenitors and Lyl- $1^{+}$microglia, in frame with the intact microglia pool in $c M y b$ deficient mice (Kierdorf et al., 2013; Schulz et al., 2012) arguing for an origin of microglia from $M \Phi^{\text {Prim }}$ rather than $M \Phi^{T-D e f}$ progenitors. Another argument comes from the zebrafish model where the fate of $\mathrm{M} \Phi^{\text {Prim }}$ progenitors is more easily traced than in mammals as they arise from a location distinct from other haematopoietic progenitors (Ferrero et al., 2018; Herbomel et al., 2001). Finally, this model is supported by the recent report that in mice lacking the ligand for cKit, the impaired development of EMPs leads to the depletion of resident MФs in the skin, lung and liver, but does not affect the development of microglia (Azzoni et al., 2018). 
Some confusion in the identification of the YS progenitors that gives rise to microglia stems from the fact that both YS waves produce cells from erythro-myeloid lineages, hence the "early EMP" and "late EMP" terminology used to qualify the brain-seeding $М \Phi$ progenitor. The monopotent/ primitive nature of E9 MФ progenitors was confirmed by our RNA-seq data, so that the $M \Phi^{\text {Prim }}$ progenitors terminology seems more appropriate than "early EMP" to qualify the primitive progenitors that give rise to microglia/BAM (Ginhoux et al., 2010; Utz et al., 2020), particularly because $M \Phi^{\text {Prim }}$ and $M \Phi^{T-D e f}$ progenitors clearly constitute distinct entities that need to be better discriminated in order to investigate their respective contribution and function in the tissues resident $\mathrm{M} \Phi$ contributed by the YS.

Amongst the features that distinguish WT E9 $M \Phi^{\text {Prim }}$ progenitors from those present at E10, the enriched expression of MHC-II and the poor expression at E9 of genes related to phagocytosis also characterise one of the two YS-derived CCR2 resident M $\Phi$ subsets identified in the heart (Epelman et al., 2014; Leid et al., 2016). A function for Lyl-1-expressing resident M $\Phi$ in cardiac development thus appears probable considering also the down-regulation of gene sets related to heart development and function in $\mathrm{E} 10 \mathrm{Lyl}-1^{\mathrm{Lacz} / L a c z} \mathrm{M} \Phi$ progenitors. This observation reinforces the need to better characterise the respective contributions of both YS-waves to resident $M \Phi$ in the tissues known to harbours YS-derived resident $M \Phi$.

By investigating Lyl-1 function at later stages of microglia development, we identified E12 and P0-P3 as key stages impacted by $L y l-1$ deficiency. These time points correspond respectively to the end of "early-" (E9-E12) and "pre"-microglia" (E14-P9) stages of the microglia development program (Matcovitch-Natan et al., 2016). Based on the gene expression pattern of Lyl-1-deficient microglia and the signature of $\mathrm{M} \Phi^{\text {Prim }}$ progenitors in the early $\mathrm{YS}$, a major contribution of $L y l-1$ deficiency to neurodevelopmental disorders may be considered. Synaptic pruning and neural maturation, which characterise the perinatal phase of microglia development (Matcovitch-Natan 


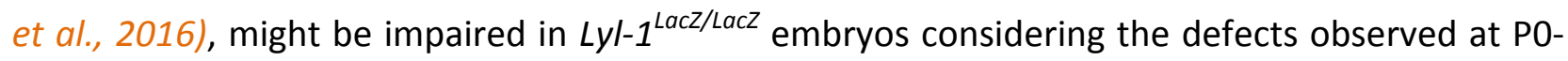
P3, the later key developmental stage regulated by Lyl-1.

Considering the sustained expression of $\mathrm{Lyl}-1$ in microglia during adulthood, and the down-

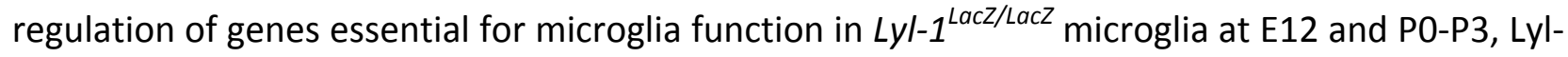
1 deregulation might contribute to various neuro-degenerative/neuro-inflammatory diseases and possibly play a role in the development of brain tumours. Such an involvement of Lyl-1 in the development of neuropathies is suggested by its deregulation in various pathological models of brain myeloid cells, uncovered through the analyses of brain myeloid cells datasets (Friedman et al., 2018) (http://research-pub.gene.com/BrainMyeloidLandscape/\#Mouse-gene/Mousegene/17095/geneReport.html). Moreover, LYL-1 expression was also reported in human microglia from healthy adult frontal cortex (Wehrspaun et al., 2015) and some reports highlight the relevance of LYL-1 deregulation in human neuro-developmental and neuro-degenerative diseases (Colangelo et al., 2002; Thomas et al., 2006). Finally, LYL-1 belongs to the 5 genes commonly deleted in patients suffering from 19p13.13 micro-deletions, which might contribute to the neuro-developmental disabilities (Nimmakayalu et al., 2013). However, since Lyl-1 is expressed in endothelial cells, including in the brain (Pirot et al., 2010), a possible contribution of $L Y L$-1-deficient endothelial cells to these diseases should be considered.

Altogether, our findings reveal Lyl-1 as a key factor regulating the production and differentiation of YS M $\Phi$ progenitors, as well as microglial development. Lyl-1 is one partners of the haematopoietic transcription factor complex which function during developmental haematopoiesis was the least studied. The development of new/more appropriate mouse models should be considered in order to carefully delineate its diverse functions in other resident $\mathrm{M} \Phi$ populations and possibly other haematopoietic and cardiovascular lineages. 


\section{MATERIALS AND METHODS}

Ethic statement. Mice were housed in the animal facility of Gustave Roussy Institute ("Plateforme d'évaluation préclinique", licence \#H94-076-11). All animal experiments were conducted in compliance with French and European laws and regulations, under authorized project \#2016030-5798, approved by officially accredited local institutional animal (committee $\mathrm{n}^{\circ} 26$ ) and French "Ministère de la Recherche" ethics boards.

Mice and embryos. We used the following mouse strains: 1- C57BL/6 mice from Harlan or Charles Rivers Laboratories, France, referred to as wild type (WT); $2-L y /-1^{\text {LacZ }}$ mice, in which an in-frame insertion of the $\beta$-Galactosidase reporter encoding gene LacZ replaced the HLH domain and the entire $3^{\prime}$ end of the $L y /-1$ gene. Lyl-1 mice were genotyped as described before (Capron et al., 2006). Lyl-1 $1^{\text {Lacz/Lacz }}$ males were crossed with WT or $L y l-1^{\text {Lacz/Lacz }}$ females to respectively generate $L y l-1^{W T / L a c z}$ or $L y l-1^{L a c z / L a c z}$ embryos. This breeding scheme avoided the possible detection of FDG/Lyl-1 expression in maternally derived M $\Phi$ (Bertrand et al., 2005b) in early Lyl$1^{\text {WT/LacZ }}$ embryos; 3-CX3cr1 $1^{\text {GFP }}$ mice (Jung et al., 2000). CX3cr1 ${ }^{\text {GFP/GFP }}$ males were crossed with C57BL/6 to generate $C \times 3 c r 1^{\text {WT/GFP }}$ mice/embryos or to $L y /-1^{\text {Lacz/LacZ }}$ females to generate CX3Cr1 ${ }^{\text {WT/GFP }}:$ Lyl-1 ${ }^{\text {WT/LacZ }}$ mice/embryos. 4-The $C \times 3 C r 1^{\text {GFP/GFP }}:$ Lyl-1 ${ }^{\text {LacZ/LacZ }}$ double mutant strain was

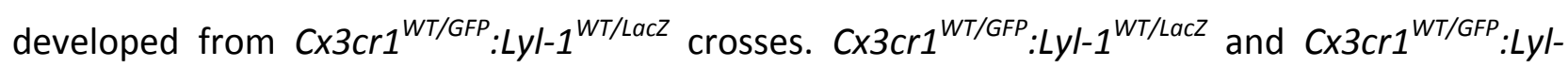
$1^{\text {Lacz/LacZ }}$ mice/embryos were obtained by crossing CX3Cr1 ${ }^{\text {GFP/GFP }}:$ Lyl-1 $1^{\text {LacZ/LacZ }}$ males to C57BL/6 or $L y \mid-1^{\text {Lacz/Lacz }}$ females, respectively. To exclude maternal/placental cell contamination, the $C \times 3 c r 1$ transgene was always inherited from the male parent.

The day of vaginal plug observation was considered as E0.5. Pregnant females were sacrificed by cervical dislocation. Pre-somite embryos were staged according to Downs et al. (Downs and Davies, 1993). E8 to E10.5 embryos were staged by somite counting and thereafter according to morphological landmarks. 
Tissues preparation and cell counts. YS (E7.5-E10.5) and E10-FL were dissected as described before (Bertrand et al., 2005a; Kieusseian et al., 2012). To perform cytometry analyses of the developing brain, two different protocols were applied:

1- For analyses performed at early stages (E9-E11), the whole brain were dissected and dissociated as previously described (Alliot et al., 1999) and further cleaned from surrounding tissues.

2- From E12 to adult stages, microglia were recovered following Percoll (P1644, Sigma) separation, according to (Mildner et al., 2007). After Percoll purification, $100 \mu \mathrm{l}$ of the cell suspension was used for cell counting and the remaining cells for flow cytometry analyses. To estimate the number of microglia per brain, the percentage of $\mathrm{CD} 11 \mathrm{~b}^{+} \mathrm{CD} 45^{\mathrm{lo}} \mathrm{F} 4 / 80^{+}$microglia was reported to the cell count recorded for the corresponding sample.

\section{In vitro culture.}

Organ culture: YS explants were placed into plates containing "Complete OptiMEM medium", i.e. OptiMEM with Glutamax (51985-042), 1\% Penicillin-streptomycin, 0.1\% $\beta$-mercaptoethanol (all from ThermoFisher) and $10 \%$ foetal calf serum (FCS; Hyclone). YS explants were maintained in organ culture at $37^{\circ} \mathrm{C}, 5 \% \mathrm{CO}_{2}$ for 1 day and are referred to as OrgD1-YS.

Clonogenic assay: Whole YS suspension or sorted cells were plated in triplicate at respectively $3 \times 10^{3}$ or $100-150$ cells $/ \mathrm{mL}$ in Methocult ${ }^{\circledR}$ M3234 (StemCell Technologies Inc.) always supplemented with Stem Cell factor $(50 \mathrm{ng} / \mathrm{mL})$, EPO $(3 \mathrm{U} / \mathrm{mL}), \quad \mathrm{IL}-3(10 \mathrm{ng} / \mathrm{mL})$, all from Peprotech, IL-6 (10 ng/mL, a gift from Sam Burstein, Maryville IL, USA), CSF-1 (10 ng/mL) and TPO (10ng/mL, provided by Kirin Brewery, Tokyo, Japan). Cultures were maintained in a humidified incubator at $37^{\circ} \mathrm{C}, 5 \% \mathrm{CO}_{2}$ and colonies were scored at day 5 for primitive erythrocytes and day 7 for the other progenitor types. 
Flow cytometry, FACS-Gal, proliferation and apoptosis assays. See Sup. table 5 for the list of antibodies and dyes used throughout this study. CD31, F4/80, CD45, CD11b and cKit antibodies were used to characterize myeloid cells. Dead cells were excluded by adding $1 \mu \mathrm{g} / \mathrm{mL}$ 7-amino actinomycin or DAPI (Sigma) before acquisition. Acquisitions were performed on a Canto II cytometer and cell sorting using FACS-Aria III or Influx (All from BD Biosciences). Data were analysed using FlowJo (Treestar) software.

FACS-Gal assay: This assay (Fiering et al., 1991; Guo and Wu, 2008), used as a reporter for Lyl-1 expression, allows the flow cytometry characterization of cells that display a $\beta$-Gal activity, using its fluorescent substrate (Fluorescein di- $\beta$-galactopyranoside (FDG) F1179; Molecular probe; Thermo Fisher).

Apoptosis assays: For apoptosis analysis, microglia were stained with anti-CD45-PECy7, antiCD11b-APC-eFluor ${ }^{\circledR} 780$ and anti-F4/80-APC, washed and incubated with Annexin V-FITC. 7AAD was added before acquisition.

Proliferation assay (BrdU incorporation): Pregnant WT and $L y l-1^{\text {Lacz/LacZ }}$ females were injected with $\mathrm{BrdU}(10 \mu \mathrm{M}) 12$ days after plug detection and sacrificed 2 hours later. Microglia were isolated and stained with CD45-PECy7, CD11b-APC-eFluor ${ }^{\circledR} 780$ and F4/80-PE antibodies. Cells were fixed, permeabilised and treated with DNase (1 hour at $\left.37^{\circ} \mathrm{C}\right)$ according to kit instruction (BD Pharmingen No. 552598) and BrdU incorporation was revealed using anti-BrdU-APC.

Brain imaging. To assess microglia morphology in E12 embryos, the midbrain was dissected from $C \times 3 c r 1^{W T / G F P}: L y /-1^{W T / W T}$ and $C \times 3 c r 1^{W T / G F P}: L y /-1^{\text {LacZ/LacZ }}$ embryos and sectioned through the midline. After fixation in $4 \%$ paraformaldehyde overnight at $4^{\circ} \mathrm{C}$, whole midbrains were washed in phosphate-buffered saline (PBS)/0.1M glycine and incubated overnight in PBS/15\% sucrose at $4^{\circ} \mathrm{C}$. Midbrains were washed with PBS $+0.1 \%$ Tween and incubated $90 \mathrm{~min}$. in blocking buffer 
(PBS+10\% FCS) at room temperature (RT). Midbrains were subsequently immuno-labelled with F4/80-APC overnight at $4^{\circ} \mathrm{C}$. After washing, they were incubated $3 \mathrm{~min}$. in PBS+DAPI $(1 \mu \mathrm{g} / \mathrm{mL})$ at RT and washed. Finally, midbrains were placed in the central well of glass-bottom culture dishes (P35G-1.5-10-C; MatTek, USA) filled with PBS+10\% FCS. After appropriate orientation of the sample, the well was covered with a $12 \mathrm{~mm} \varnothing$ glass coverslip. Image stacks were collected using a Leica SP8 confocal microscope. Images were processed using Imaris x 64 (version 7.7.2; Bitplane) and Photoshop 8.0 (Adobe Systems, San Jose, CA) softwares. To unsure an unbiased choice of the cells imaged, taking into account possible changes in cell distribution induced by Lyl-1 deficiency, we always acquired cells in similar positions regarding the landmark set in the midbrain flat mount, as shown in Sup. fig. 4c.

RT-qPCR analyses. Total RNA was extracted from sorted $\mathrm{CD} 11 \mathrm{~b}^{+} \mathrm{F} 4 / 80^{+} \mathrm{CD} 45^{\text {low }}$ microglia using Trizol (ThermoFisher). After cDNA synthesis using a SuperScript ${ }^{\mathrm{TM}}$ VILO $^{\mathrm{TM}}$ Master Mix reverse transcriptase (ThermoFisher), quantitative PCR was performed using SYBR Premix Ex Taqll (Tli RNase H Plus, Takara Bio). Reference genes were Actin, Hprt and Tubulin. Gene expressions were

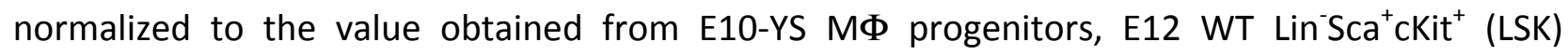
progenitors or E12 WT microglia, and relative gene expression levels were determined by the $\Delta \Delta \mathrm{Ct}$ method. Gene expression was considered undetectable if $\mathrm{Ct}$ values were $>35$ cycles. The sequences of the primers used are provided in Sup. table 6.

\section{RNA-sequencing.}

Sample preparation: $M \Phi$ progenitors $\left(\mathrm{CD} 45^{+} \mathrm{CD} 11 \mathrm{~b}^{+} \mathrm{cKit}{ }^{+}\right)$were sorted from $\mathrm{E9}\left(\mathrm{M} \Phi^{\text {Prim }}\right.$ progenitor) and $\mathrm{E} 10\left(\mathrm{M} \Phi^{\text {Prim }}+\mathrm{M} \Phi^{\mathrm{T}-\mathrm{Def}}\right.$ progenitors) YS pools from either WT or $L y / \mathbf{1}^{\text {LacZ/LacZ }}$ 
embryos. Four biological replicates were prepared for each sample. RNA was extracted as described above.

Sample processing: The RNA integrity (RNA Integrity Score $\geq 7.0$ ) was checked on the Agilent 2100 Bioanalyzer (Agilent) and quantity was determined using Qubit (Invitrogen). SureSelect Automated Strand Specific RNA Library Preparation Kit was used according to manufacturer's instructions with the Bravo Platform. Briefly, 50ng of total RNA sample was used for poly-A mRNA selection using oligo(dT) beads and subjected to thermal mRNA fragmentation. The fragmented mRNA samples were subjected to cDNA synthesis and further converted into double stranded DNA using the reagents supplied in the kit, and the resulting dsDNA was used for library preparation. The final libraries were bar-coded, purified, pooled together in equal concentrations and subjected to paired-end sequencing $(2 \times 100)$ on Novaseq-6000 sequencer (Illumina) at Gustave Roussy genomic facility. RNA-seq data have been deposited in the ArrayExpress database at EMBL-EBI (www.ebi.ac.uk/arrayexpress) under accession number E-MTAB-9618.

RNA-sequencing analysis: Quality of RNA-seq reads was assessed with FastQC 0.11 .7 and MultiQC 1.5 (Ewels et al., 2016). Low quality reads were trimmed with Trimmomatic 0.33 (Bolger et al., 2014). Salmon 0.9.0 tool (Patro et al., 2017) was used for quantifying the expression of transcripts using geneset annotation from Gencode project release M17 for mouse (Frankish et al., 2019). The version of transcriptome reference sequences used was GRCm38.p6.

Statistical analysis was performed using $\mathrm{R}$ with the method proposed by Anders and Huber implemented in the DESeq2 Bioconductor package (Love et al., 2014). The differential expression analysis in DESeq2 uses a generalized linear model (GLM) where counts are modelled using a negative binomial distribution. Counts were normalized from the estimated size factors using the median ratio method and a Wald test was used to test the significance of GLM coefficients. 
Genes have been considered differentially expressed when adjusted $p$-value $<0.05$ and foldchange $>2$.

Data were analysed through the use of Ingenuity ${ }^{\circledR}$ Pathway Analysis (QIAGEN Inc., https://www.qiagenbioinformatics.com/products/ingenuity-pathway-analysis) (Krämer et al., 2014), Gene set enrichment analysis (GSEA; https://www.gsea-msigdb.org/gsea/index.jsp) (Mootha et al., 2003; Subramanian et al., 2005), Morpheus (https://software.broadinstitute.org/morpheus/) and Venny (https://bioinfogp.cnb.csic.es/tools/venny/) softwares.

Statistical analysis. Statistical tests were performed using Prism 7 (GraphPad) Software. Statistical significance is indicated by the exact $p$-value and/or as $* p<0.05, * * p<0.01, * * *<$ 0.001 and ${ }^{* * * *} \mathrm{p}<0.0001$ 


\section{Acknowledgements.}

The authors thank Julien Bertrand for critical reading of the manuscript. We are grateful to the staff of the facilities at Gustave Roussy, the animal facility (PFEP, UMS AMMICa UMS 3655/US23, directed by P. Gonin), the imaging facility (PFIC, UMS AMMICa UMS 3655/US23, directed by C. Laplace-Builhe), the genomic facility directed by N. Droin, the bioinformatics facility (G. Meurice), directed by M. Deloger.

This work was supported by fundings from Institut National de la Santé et de la Recherche Médicale to W. Vainchenker, I. Plo and H. Raslova, from Centre National de la Recherche Scientifique and Université de Paris-Saclay to I. Godin, from grants INCA PLBio to I. Plo, "Ligue Nationale contre le Cancer" Certified Team to H. Raslova, "Association pour la Recherche sur le Cancer" ( ${ }^{\circ} 4878$ ) to I. Godin, Gustave Roussy (TA DERE 17) to D. Ren, Grant Agency of the Czech Republic (GACR $\mathrm{n}^{\circ} 19-23154 S$ ) to D. Filipp and from fellowships from "Association pour la Recherche sur le Cancer" to A.-L. Kaushik; "Société Française d'Hématologie" to S. Wang and Chinese Scolarship Council fellowships to S. Wang and D. Ren.

\section{Author Information.}

The authors declare no competing financial interests.

Correspondence should be addressed to I.G. (Isabelle.Godin@gustaveroussy.fr). 


\section{REFERENCES}

Alliot, F., Godin, I., and Pessac, B. (1999). Microglia derive from progenitors, originating from the yolk sac, and which proliferate in the brain. Brain Res Dev Brain Res 117, 145-152.

Antony, J.M., Paquin, A., Nutt, S.L., Kaplan, D.R., and Miller, F.D. (2011). Endogenous microglia regulate development of embryonic cortical precursor cells. J Neurosci Res 89, 286-298.

Azzoni, E., Frontera, V., McGrath, K.E., Harman, J., Carrelha, J., Nerlov, C., Palis, J., Jacobsen, S.E.W., and de Bruijn, M.F.T.R. (2018). Kit ligand has a critical role in mouse yolk sac and aorta-gonadmesonephros hematopoiesis. EMBO reports.

Balounova, J., Splichalova, I., Dobesova, M., Kolar, M., Fiser, K., Prochazka, J., Sedlacek, R., Jurisicova, A., Sung, H.K., Korinek, V., et al. (2019). Toll-like receptor 2 expression on c-kit(+) cells tracks the emergence of embryonic definitive hematopoietic progenitors. Nat Commun 10, 5176.

Bertrand, J.Y., Giroux, S., Cumano, A., and Godin, I. (2005a). Hematopoietic stem cell development during mouse embryogenesis. Methods Mol Med 105, 273-288.

Bertrand, J.Y., Jalil, A., Klaine, M., Jung, S., Cumano, A., and Godin, I. (2005b). Three pathways to mature macrophages in the early mouse yolk sac. Blood 106, 3004-3011.

Bisht, K., Sharma, K., and Tremblay, M.-È. (2018). Chronic stress as a risk factor for Alzheimer's disease: Roles of microglia-mediated synaptic remodeling, inflammation, and oxidative stress. Neurobiology of Stress 9, 9-21.

Bolger, A.M., Lohse, M., and Usadel, B. (2014). Trimmomatic: a flexible trimmer for Illumina sequence data. Bioinformatics 30, 2114-2120.

Capron, C., Lécluse, Y., Kaushik, A.L., Foudi, A., Lacout, C., Sekkai, D., Godin, I., Albagli, O., Poullion, I., Svinartchouk, F., et al. (2006). The SCL relative LYL-1 is required for fetal and adult hematopoietic stem cell function and B-cell differentiation. Blood 107, 4678-4686.

Chiu, S.K., Orive, S.L., Moon, M.J., Saw, J., Ellis, S., Kile, B.T., Huang, Y., Chacon, D., Pimanda, J.E., Beck, D., et al. (2019). Shared roles for Scl and Lyl1 in murine platelet production and function. Blood 134, 826835.

Colangelo, V., Schurr, J., Ball, M.J., Pelaez, R.P., Bazan, N.G., and Lukiw, W.J. (2002). Gene expression profiling of 12633 genes in Alzheimer hippocampal CA1: transcription and neurotrophic factor downregulation and up-regulation of apoptotic and pro-inflammatory signaling. J Neurosci Res 70, 462-473.

Crotti, A., and Ransohoff, R.M. (2016). Microglial Physiology and Pathophysiology: Insights from Genome-wide Transcriptional Profiling. Immunity 44, 505-515.

Cumano, A., Dieterlen-Lievre, F., and Godin, I. (1996). Lymphoid potential, probed before circulation in mouse, is restricted to caudal intraembryonic splanchnopleura. Cell 86, 907-916. 
Cumano, A., Ferraz, J.C., Klaine, M., Di Santo, J.P., and Godin, I. (2001). Intraembryonic, but not yolk sac hematopoietic precursors, isolated before circulation, provide long-term multilineage reconstitution. Immunity 15, 477-485.

Cumano, A., and Godin, I. (2007). Ontogeny of the hematopoietic system. Annu Rev Immunol 25, 745785.

Curtis, D.J., Salmon, J.M., and Pimanda, J.E. (2012). Concise Review: Blood Relatives: Formation and regulation of hematopoietic stem cells by the basic helix-loop-helix transcription factors stem cell leukemia and lymphoblastic leukemia-derived sequence 1. Stem Cells 30, 1053-1058.

Downs, K.M., and Davies, T. (1993). Staging of gastrulating mouse embryos by morphological landmarks in the dissecting microscope. Development 118, 1255-1266.

Epelman, S., Lavine, K.J., Beaudin, A.E., Sojka, D.K., Carrero, J.A., Calderon, B., Brija, T., Gautier, E.L., Ivanov, S., Satpathy, A.T., et al. (2014). Embryonic and Adult-Derived Resident Cardiac Macrophages Are Maintained through Distinct Mechanisms at Steady State and during Inflammation. Immunity 40, 91-104.

Ewels, P., Magnusson, M., Lundin, S., and Käller, M. (2016). MultiQC: summarize analysis results for multiple tools and samples in a single report. Bioinformatics 32, 3047-3048.

Ferrero, G., Mahony, C.B., Dupuis, E.o., Yvernogeau, L., Di Ruggiero, E., Miserocchi, M., Caron, M., Robin, C., Traver, D., Bertrand, J.Y., et al. (2018). Embryonic Microglia Derive from Primitive Macrophages and Are Replaced by cmyb-Dependent Definitive Microglia in Zebrafish. Cell Reports 24, 130-141.

Ferrier, R., Nougarede, R., Doucet, S., Kahn-Perles, B., Imbert, J., and Mathieu-Mahul, D. (1999). Physical interaction of the bHLH LYL1 protein and NF-kappaB1 p105. Oncogene 18, 995-1005.

Fiering, S.N., Roederer, M., Nolan, G.P., Micklem, D.R., Parks, D.R., and Herzenberg, L.A. (1991). Improved FACS-Gal: flow cytometric analysis and sorting of viable eukaryotic cells expressing reporter gene constructs. Cytometry 12, 291-301.

Frankish, A., Diekhans, M., Ferreira, A.M., Johnson, R., Jungreis, I., Loveland, J., Mudge, J.M., Sisu, C., Wright, J., Armstrong, J., et al. (2019). GENCODE reference annotation for the human and mouse genomes. Nucleic Acids Res 47, D766-d773.

Friedman, B.A., Srinivasan, K., Ayalon, G., Meilandt, W.J., Lin, H., Huntley, M.A., Cao, Y., Lee, S.-H., Haddick, P.C.G., Ngu, H., et al. (2018). Diverse Brain Myeloid Expression Profiles Reveal Distinct Microglial Activation States and Aspects of Alzheimer's Disease Not Evident in Mouse Models. Cell Reports 22, 832847.

Ginhoux, F., Greter, M., Leboeuf, M., Nandi, S., See, P., Gokhan, S., Mehler, M.F., Conway, S.J., Ng, L.G., Stanley, E.R., et al. (2010). Fate Mapping Analysis Reveals That Adult Microglia Derive from Primitive Macrophages. Science 330, 841-845.

Ginhoux, F., and Guilliams, M. (2016). Tissue-Resident Macrophage Ontogeny and Homeostasis. Immunity 44, 439-449. 
Giroux, S., Kaushik, A.L., Capron, C., Jalil, A., Kelaidi, C., Sablitzky, F., Dumenil, D., Albagli, O., and Godin, I. (2007). Iyl-1 and tal-1/scl, two genes encoding closely related bHLH transcription factors, display highly overlapping expression patterns during cardiovascular and hematopoietic ontogeny. Gene Expr Patterns $7,215-226$

Goldmann, T., Wieghofer, P., Jordão, M.J.C., Prutek, F., Hagemeyer, N., Frenzel, K., Amann, L., Staszewski, O., Kierdorf, K., Krueger, M., et al. (2016). Origin, fate and dynamics of macrophages at central nervous system interfaces. Nature Immunology 17, 797-805.

Gomez Perdiguero, E., Klapproth, K., Schulz, C., Busch, K., Azzoni, E., Crozet, L., Garner, H., Trouillet, C., de Bruijn, M.F., Geissmann, F., et al. (2015). Tissue-resident macrophages originate from yolk-sac-derived erythro-myeloid progenitors. Nature 518, 547-551.

Guo, W., and Wu, H. (2008). Detection of LacZ expression by FACS-Gal analysis. Nature Protocol Exchange

Hagemeyer, N., Kierdorf, K., Frenzel, K., Xue, J., Ringelhan, M., Abdullah, Z., Godin, I., Wieghofer, P., Costa Jordao, M.J., Ulas, T., et al. (2016). Transcriptome-based profiling of yolk sac-derived macrophages reveals a role for Irf8 in macrophage maturation. EMBO J 35, 1730-1744.

Herbomel, P., Thisse, B., and Thisse, C. (2001). Zebrafish early macrophages colonize cephalic mesenchyme and developing brain, retina, and epidermis through a M-CSF receptor-dependent invasive process. Dev Biol 238, 274-288.

Hoeffel, G., Chen, J., Lavin, Y., Low, D., Almeida, F.F., See, P., Beaudin, A.E., Lum, J., Low, I., Forsberg, E.C., et al. (2015). C-Myb+ Erythro-Myeloid Progenitor-Derived Fetal Monocytes Give Rise to Adult TissueResident Macrophages. Immunity 42, 665-678.

Hoeffel, G., and Ginhoux, F. (2018). Fetal monocytes and the origins of tissue-resident macrophages. Cell Immunol 330, 5-15.

Jung, S., Aliberti, J., Graemmel, P., Sunshine, M.J., Kreutzberg, G.W., Sher, A., and Littman, D.R. (2000). Analysis of fractalkine receptor CX(3)CR1 function by targeted deletion and green fluorescent protein reporter gene insertion. Mol Cell Biol 20, 4106-4114.

Kierdorf, K., Erny, D., Goldmann, T., Sander, V., Schulz, C., Perdiguero, E.G., Wieghofer, P., Heinrich, A., Riemke, P., Holscher, C., et al. (2013). Microglia emerge from erythromyeloid precursors via Pu.1- and Irf8-dependent pathways. Nat Neurosci 16, 273-280.

Kieusseian, A., Brunet de la Grange, P., Burlen-Defranoux, O., Godin, I., and Cumano, A. (2012). Immature hematopoietic stem cells undergo maturation in the fetal liver. Development 139, 3521-3530.

Krämer, A., Green, J., Pollard, J., Jr., and Tugendreich, S. (2014). Causal analysis approaches in Ingenuity Pathway Analysis. Bioinformatics 30, 523-530.

Lavin, Y., Winter, D., Blecher-Gonen, R., David, E., Keren-Shaul, H., Merad, M., Jung, S., and Amit, I. (2014). Tissue-Resident Macrophage Enhancer Landscapes Are Shaped by the Local Microenvironment. Cell 159, 1312-1326. 
Leid, J., Carrelha, J., Boukarabila, H., Epelman, S., Jacobsen, S.E.W., and Lavine, K.J. (2016). Primitive Embryonic Macrophages are Required for Coronary Development and Maturation. Circulation Research $118,1498$.

Love, M.I., Huber, W., and Anders, S. (2014). Moderated estimation of fold change and dispersion for RNA-seq data with DESeq2. Genome Biol 15, 550.

Mass, E. (2018). Delineating the origins, developmental programs and homeostatic functions of tissueresident macrophages. Int Immunol 30, 493-501.

Mass, E., Ballesteros, I., Farlik, M., Halbritter, F., Gunther, P., Crozet, L., Jacome-Galarza, C.E., Handler, K., Klughammer, J., Kobayashi, Y., et al. (2016). Specification of tissue-resident macrophages during organogenesis. Science 353.

Matcovitch-Natan, O., Winter, D.R., Giladi, A., Vargas Aguilar, S., Spinrad, A., Sarrazin, S., Ben-Yehuda, H., David, E., Zelada González, F., Perrin, P., et al. (2016). Microglia development follows a stepwise program to regulate brain homeostasis. Science 353, aad8670.

McCormack, M.P., Shields, B.J., Jackson, J.T., Nasa, C., Shi, W., Slater, N.J., Tremblay, C.S., Rabbitts, T.H., and Curtis, D.J. (2013). Requirement for Lyl1 in a model of Lmo2-driven early T-cell precursor ALL. Blood 122, 2093-2103.

McGrath, K.E., Frame, J.M., and Palis, J. (2015). Early hematopoiesis and macrophage development. Seminars in Immunology 27, 379-387.

Mildner, A., Schmidt, H., Nitsche, M., Merkler, D., Hanisch, U.K., Mack, M., Heikenwalder, M., Bruck, W., Priller, J., and Prinz, M. (2007). Microglia in the adult brain arise from Ly-6ChiCCR2+ monocytes only under defined host conditions. Nat Neurosci 10, 1544-1553.

Mootha, V.K., Lindgren, C.M., Eriksson, K.F., Subramanian, A., Sihag, S., Lehar, J., Puigserver, P., Carlsson, E., Ridderstråle, M., Laurila, E., et al. (2003). PGC-1alpha-responsive genes involved in oxidative phosphorylation are coordinately downregulated in human diabetes. Nat Genet 34, 267-273.

Nimmakayalu, M., Horton, V.K., Darbro, B., Patil, S.R., Alsayouf, H., Keppler-Noreuil, K., and Shchelochkov, O.A. (2013). Apparent germline mosaicism for a novel 19p13.13 deletion disrupting NFIX and CACNA1A. Am J Med Genet A 161A, 1105-1109.

Palis, J. (2016). Hematopoietic stem cell-independent hematopoiesis: emergence of erythroid, megakaryocyte, and myeloid potential in the mammalian embryo. FEBS Lett 590, 3965-3974.

Palis, J., Robertson, S., Kennedy, M., Wall, C., and Keller, G. (1999). Development of erythroid and myeloid progenitors in the yolk sac and embryo proper of the mouse. Development 126, 5073-5084.

Palpant, N.J., Wang, Y., Hadland, B., Zaunbrecher, R.J., Redd, M., Jones, D., Pabon, L., Jain, R., Epstein, J., Ruzzo, W.L., et al. (2017). Chromatin and Transcriptional Analysis of Mesoderm Progenitor Cells Identifies HOPX as a Regulator of Primitive Hematopoiesis. Cell Reports 20, 1597-1608.

Patro, R., Duggal, G., Love, M.I., Irizarry, R.A., and Kingsford, C. (2017). Salmon provides fast and biasaware quantification of transcript expression. Nat Methods 14, 417-419. 
Pina, C., and Enver, T. (2007). Differential contributions of haematopoietic stem cells to foetal and adult haematopoiesis: insights from functional analysis of transcriptional regulators. Oncogene 26, 67506765.

Pirot, N., Deleuze, V., El-Hajj, R., Dohet, C., Sablitzky, F., Couttet, P., Mathieu, D., and Pinet, V. (2010). LYL1 activity is required for the maturation of newly formed blood vessels in adulthood. Blood 115, 52705279.

Porcher, C., Chagraoui, H., and Kristiansen, M.S. (2017). SCL/TAL1: a multifaceted regulator from blood development to disease. Blood 129, 2051-2060.

Schulz, C., Gomez Perdiguero, E., Chorro, L., Szabo-Rogers, H., Cagnard, N., Kierdorf, K., Prinz, M., Wu, B., Jacobsen, S.E., Pollard, J.W., et al. (2012). A lineage of myeloid cells independent of Myb and hematopoietic stem cells. Science 336, 86-90.

Senserrich, J., Batsivari, A., Rybtsov, S., Gordon-Keylock, S., Souilhol, C.I., Buchholz, F., Hills, D., Zhao, S., and Medvinsky, A. (2018). Analysis of Runx1 Using Induced Gene Ablation Reveals Its Essential Role in Pre-liver HSC Development and Limitations of an In Vivo Approach, Vol 11.

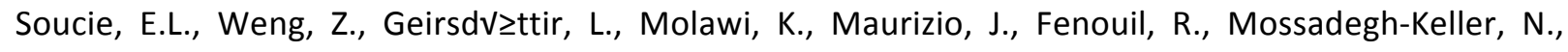
Gimenez, G., VanHille, L., Beniazza, M., et al. (2016). Lineage-specific enhancers activate self-renewal genes in macrophages and embryonic stem cells. Science 351.

Subramanian, A., Tamayo, P., Mootha, V.K., Mukherjee, S., Ebert, B.L., Gillette, M.A., Paulovich, A., Pomeroy, S.L., Golub, T.R., Lander, E.S., et al. (2005). Gene set enrichment analysis: a knowledge-based approach for interpreting genome-wide expression profiles. Proc Natl Acad Sci U S A 102, 15545-15550.

Thomas, D.M., Francescutti-Verbeem, D.M., and Kuhn, D.M. (2006). Gene expression profile of activated microglia under conditions associated with dopamine neuronal damage. Faseb J 20, 515-517.

Ueno, M., Fujita, Y., Tanaka, T., Nakamura, Y., Kikuta, J., Ishii, M., and Yamashita, T. (2013). Layer V cortical neurons require microglial support for survival during postnatal development. Nat Neurosci 16 , 543-551.

Utz, S.G., See, P., Mildenberger, W., Thion, M.S., Silvin, A., Lutz, M., Ingelfinger, F., Rayan, N.A., Lelios, I., Buttgereit, A., et al. (2020). Early Fate Defines Microglia and Non-parenchymal Brain Macrophage Development. Cell 181, 557-573 e518.

Wehrspaun, C.C., Haerty, W., and Ponting, C.P. (2015). Microglia recapitulate a hematopoietic master regulator network in the aging human frontal cortex. Neurobiol Aging 36, 2443 e2449-2443 e2420.

Wilson, N.K., Foster, S.D., Wang, X., Knezevic, K., Sch,tte, J., Kaimakis, P., Chilarska, P.M., Kinston, S., Ouwehand, W.H., Dzierzak, E., et al. (2010). Combinatorial Transcriptional Control In Blood Stem/Progenitor Cells: Genome-wide Analysis of Ten Major Transcriptional Regulators. Cell Stem Cell 7, 532-544.

Wittamer, V., and Bertrand, J.Y. (2020). Yolk sac hematopoiesis: does it contribute to the adult hematopoietic system? Cell Mol Life Sci. 


\section{FIGURE LEGENDS}

\section{Figure 1: Lyl-1 expression discriminates $M \Phi^{\text {Prim }}$ from $M \Phi^{T-D e f}$ progenitors in the early YS}

a. Lyl-1 deficiency leads to an increased production of $M \Phi$ progenitors in the early YS: Left: Clonogenic

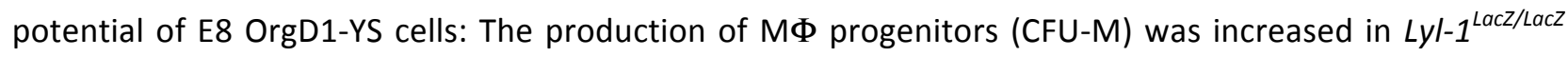
OrgD1-YS ( $n=3-5$, each sample containing 3-6 YS; mean \pm s.e.m.; Unpaired, two-tailed $t$-Test). The size of the $\mathrm{M} \Phi$ colonies and the cell morphology were similar for the 3 genotypes (data not shown). Right: The distribution of other progenitors with a myeloid potential (EMP and GM) was similar in WT, Lyl-1 ${ }^{\text {WT/Lacz }}$ and $L y l-1^{\text {Lacz/LacZ }}$ E8 OrgD1-YS.

b. While all MФ progenitors in E9-YS (left panel) expressed FDG/Lyl-1, E9.5 and E10-YS (middle panels) harboured two MФ progenitors subsets discriminated by their FDG/Lyl-1 expression. FDG ${ }^{+} /$Lyl- $^{+}$and FDG

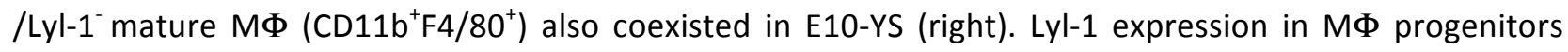
was analysed by FACS-Gal assay, using the $\beta$-Gal fluorescent substrate FDG as a reporter for Lyl-1 expression. The contour plots in WT samples indicate the level of non-specific background $\beta$-Gal activity/FDG labelling in WT samples. Representative profiles of 3 independent samples, each consisting of 3-4 YS (See the gating strategy in Sup. fig. 1a).

c. $\mathbf{M} \Phi^{\text {Prim }}$ progenitors express Lyl-1. Upper panel: Flow cytometry profiles of WT (left) and Lyl-1 $1^{W T / L a c z}$

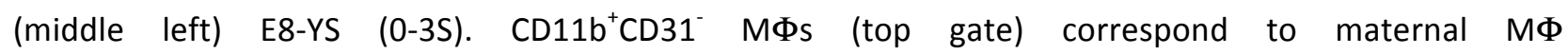
present at this early stage (Bertrand et al., 2005). All CD11b $\mathrm{CD}^{+} 1^{+} \mathrm{M} \Phi$ progenitors (lower gate) displayed FDG/Lyl-1 expression.

d. FDG/Lyl-1 positive and negative myeloid progenitors produce a distinct progeny: The type of progenitors produced by sorted Ter119-cKit ${ }^{+} \mathrm{CD} 45^{+} \mathrm{CD} 11 \mathrm{~b}^{+}$myeloid progenitors was determined by clonogenic assays using E9 WT and $L y l-1^{W T / L a c Z}$ YS $(<18 \mathrm{~S} ; \mathrm{n}=7)$, and E10 WT YS (n=15) in 3 independent experiments. At E10, myeloid progenitors from $L y /-1^{W T / L a c Z}$ YS were subdivided into FDG/Lyl-1 negative $(n=15)$ and positive $(n=12)$ fractions (5 independent experiments). Samples were biological replicates comprising 6-8 YS. 100 to $150 \mathrm{cKit}^{+} \mathrm{CD} 45^{+} \mathrm{CD} 11 \mathrm{~b}^{+}$cells per condition were platted in triplicate. $\mathrm{FDG}^{+} / \mathrm{Lyl}^{+} 1^{+}$ progenitors essentially produced $\mathrm{M} \Phi$ colonies, while FDG $/$ Lyl-1' progenitors produced also GM and G colonies, thus belonging to the transient definitive wave.

e. RT-qPCR quantification of $c M y b$ expression levels in $c \mathrm{Kit}^{+} \mathrm{CD} 45^{+} \mathrm{CD} 11 \mathrm{~b}^{+} \mathrm{M} \Phi$ progenitors sorted from WT E9-YS, WT and Lyl-1 ${ }^{\text {WT/LacZ }}$ E10-YS, as well as from the FDG/Lyl-1 positive and negative fractions of M $\Phi$ progenitors from $L y /-1^{W T / L a c Z}$ E10-YS. Lin Sca ${ }^{+} \mathrm{CKit}^{+}$(LSK) progenitors from WT E12 FL were used as positive control. FDG ${ }^{+} / \mathrm{Lyl}-1^{+} \mathrm{M} \Phi$ progenitors from E10-YS expressed $c M y b^{\text {Low/Neg }}$ levels similar to E9-YS, which characterize the primitive YS wave. The FDG /Lyl-1- fraction expressed significantly higher $c M y b$ levels, similar to LSK cells from E12-FL. cMyb expression levels, shown on a $\log ^{2}$ scale, were normalized to the mean expression value obtained for WT E10-YS, considered as 1 (Unpaired, two-tailed $t$-Test). 


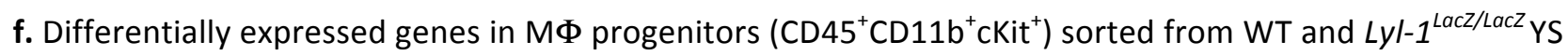
at E9 and E10. Upper panel: Unsupervised principal component analysis (PCA) plot positioned E9 and E10 $M \Phi$ progenitors in two distinct groups, followed by segregation of WT and $L y /-1^{\text {Lacz/LacZ }}$ samples. Lower panel: Volcano plot of E9 WT vs E10 WT M $\Phi$ progenitors. Red and green dots indicate genes with statistically significant changes in expression level. ( $p$-value $<0.05$, absolute fold change $\geq 2.0$ ) (NDE: not deregulated genes; DE-Up: up-regulated genes; DE-Down: down-regulated genes).

g. Upper panel: Venn diagram comparing DEGs in E9 WT versus E10 WT M $\Phi$ progenitors to the EMP (Left)

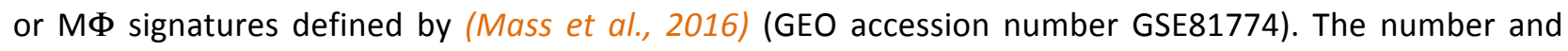
percentage of DEGs common to the EMP or $M \Phi$ signatures is shown.

Lower panel: Expression profiles of the overlapping genes identified by the Venn diagram (Heatmap displays transformed log2-expression values; Unpaired $t$-Test, two-tailed). Note the higher expression at E10 of genes involved in erythroid (Haemoglobins: Pink arrow; Transcription factors: green arrow), as well as megakaryocytic and granulocytic-related genes (blue arrow).

h. Relative expression levels of Gata1 and Spi1/PU.1, as indicated by their relative Transcripts per million kilo-bases (TPM).

i. Enriched Pathways in E9 and E10 WT MF progenitors with absolute z-score $\geq 2$, from QIAGEN's Ingenuity ${ }^{\circledR}$ Pathway Analysis (IPA). Bars: minus log of the $p$-value of each canonical pathway; Orange line: threshold $p$-value of 0.05. Ratio: genes detected/genes per pathway.

j. Expression profiles of DEGs related to IFNY and IFN $\beta$ response, identified by g:Profiler. (Heatmap displays transformed log2-expression values; unpaired t-Test, two-tailed).

k. Expression profiles of DEGs related to MHC-II complex (Heatmap displays transformed log2-expression values; unpaired $t$-Test, two-tailed).

I. Expression profiles of DEGs related to cytokine signalling (Heatmap displays transformed log2expression values; unpaired $t$-Test, two-tailed).

\section{Figure 2:}

\section{a. Lyl-1 regulates the production of E8 $M \Phi^{\text {Prim }}$ progenitors.}

Left panel: Flow cytometry quantification of $\mathrm{FDG}^{+} / \mathrm{Lyl}-1^{+} \mathrm{CD} 11 \mathrm{~b}^{+} \mathrm{CD} 31^{+} \mathrm{M} \Phi$ progenitors from WT and Lyl-

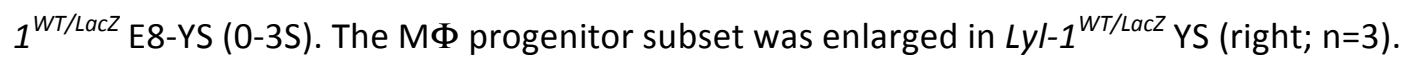

Right panel: In clonogenic assays, less than one EMP and/or GM progenitor per E8-YS was detected in WT and mutant samples, confirming that the assay was performed at a time when EMP-derived$M \Phi$ progenitors were absent. The majority of the $25-30$ colonies per YS were Ery ${ }^{p}$ (60 to $80 \%$ in the 3 genotypes). The number of M $\Phi$ colonies obtained from E8-YS (0-3S) was increased in Lyl-1 ${ }^{W T / L a c z}$ and Lyl$1^{\text {Lacz/Lacz }}$ compared to WT (left). Other progenitors were occasionally and randomly found in the Lyl-1 WT/Lacz 
$\mathrm{FDG}^{+} / \mathrm{Lyl}^{-1^{+}}$fraction including EMPs $(0.81 \% \pm 0.66 ; n=3)$. ( $n=3-5$, each sample contained $5-10$ YS; plots show mean \pm s.e.m.; Unpaired, two-tailed $t$-Test).

b. The relative expression levels (read counts) of the CD41 coding gene Itg2b is increased in Lyl-1/acz/lacz M $\Phi$ progenitors compared to WT at E9 (unpaired $t$-Test, two-tailed).

c. Relative expression levels of transcriptions factors regulating haematopoietic progenitor emergence in Lyl-1 ${ }^{\text {Lacz/Lacz }} \mathrm{M} \Phi$ progenitors compared to WT at E9 ( $p$-values were determined by unpaired, two-tailed $t$ Test).

d. GSEA pathways (Top; FDR q-Value <0.29) and GO terms (Bottom; FDR q-value <0.01) enriched in E9 Lyl$1^{\text {lacz/lacz }}$ compared to E9 WT MФ progenitors. Highlighted are the pathways specifically related to embryo patterning (blue) and to the development of skeletal (green) and nervous systems (yellow). Pink arrows point to changes related to metabolic pathways.

Figure 3: The differentiation $M \Phi$ progenitors is defective in $L y /-1^{\text {Lacz/Lacz }} Y S$.

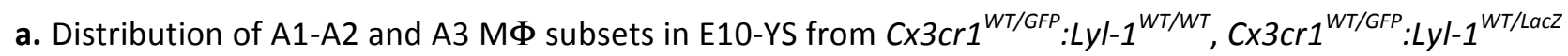

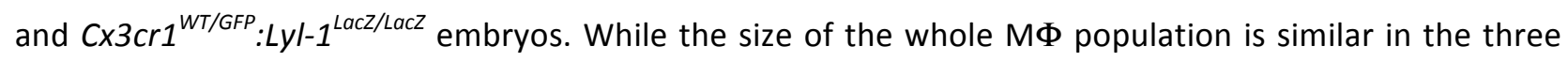
genotypes (Top panel), $L y l-1$ deficiency leads to a modified distribution of the $M \Phi$ subsets (middle and lower panel) with an increased size of the $A 1$ subset and a reduced $A 3$ pool (5-12 independent analyses, each sample cumulating 6-8 YS. Plots show mean \pm s.e.m.; Unpaired, two-tailed $t$-Test).

b. GSEA pathway indicates a deficit in Jak1-Stat signalling in $L y /-1^{\text {Lacz/Lacz }} M \Phi$ progenitors compared to WT at E9 (NES: normalised enrichment score; FDR: false discovery rate).

c. Relative expression levels (read counts) of haematopoietic markers in WT and $L y /-1^{\text {Lacz/LacZ } M \Phi}$ progenitors at E9 (unpaired $t$-Test, two-tailed).

d. Top 1 GSEA pathway indicates that the IFN signalling pathway (left) which characterize E9 $M \Phi^{\text {Prim }}$ progenitors, and particularly Irf8 (right), is defective in Lyl-1 ${ }^{\text {Lacz/LacZ }} \mathrm{M} \Phi$ progenitors (NES: normalised enrichment score; FDR: false discovery rate).

e. From the 53 canonical pathways identified by IPA in the DEGs, 9 were enriched with an absolute $Z$ score 21. Bars: minus log of the $p$-value of each canonical pathway; Orange line: threshold $p$-value of 0.05 . Ratio: genes detected/genes per pathway.

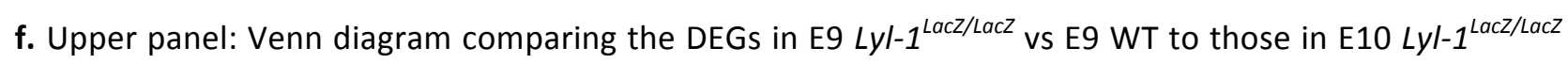
vs E10 WT MФ progenitors. Lower panel: Expression profiles of the DEGs common to both stages identified by the Venn comparison (Heatmap displays transformed log2-expression values; unpaired $t$ Test, two-tailed). 


\section{Figure 4:}

a. Left panel: All M $\Phi$ progenitors from E10-Brain (Left) expressed Lyl-1, contrary to the corresponding YS (Fig. $1 \mathrm{~b}$ right) which harbour both $\mathrm{FDG}^{+} / \mathrm{Lyl}^{-1^{+}}$and $\mathrm{FDG} / \mathrm{Lyl}-1^{-}$subsets. M $\Phi$ progenitors from $\mathrm{E} 10 \mathrm{Lyl}-$

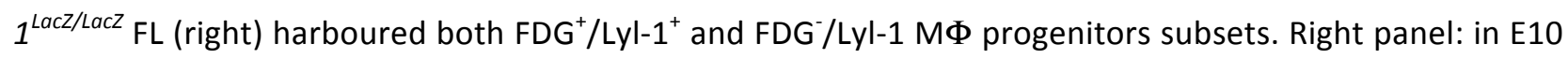
brain, mature $\mathrm{M} \Phi\left(\mathrm{CD} 11 \mathrm{~b}^{+} \mathrm{F} 4 / 80^{+}\right.$gate) were all FDG ${ }^{+} / \mathrm{Lyl}^{-} \mathrm{1}^{+}$

The contour plots in WT samples indicate the level of non-specific background $\beta$-Gal activity/FDG labelling in WT samples. Representative profiles of 3 independent samples, each consisting of 3-4 Brain or 8-12 E10-FL.

b. Quantification of $\mathrm{CKit}^{+} \mathrm{CD} 45^{+} \mathrm{CD} 11 \mathrm{~b}^{+} \mathrm{M} \Phi$ progenitors in E10-FL (plots show mean \pm s.e.m.; Unpaired, two-tailed $t$-Test).

c. Lyl-1 marks the entire F4/80+ microglia/BAM population from the onset of brain colonisation. The rare $\mathrm{CD} 11 \mathrm{~b}+\mathrm{F} 4 / 80^{\text {low-neg }}$ cells present in the brain at $\mathrm{E9}$ are FDG/Lyl-positive (Top Panel). Grey histograms indicate non-specific background $\beta$-Gal activity/FDG levels in WT samples.

d. $M \Phi$ progenitors from E10 brain expressed $c M y b$ levels similar to E9-YS $M \Phi^{\text {Prim }}$ progenitors. RT-qPCR quantification of $c M y b$ expression levels in $\mathrm{CKit}^{+} \mathrm{CD} 45^{+} \mathrm{CD} 11 \mathrm{~b}^{+} \mathrm{M} \Phi$ progenitors sorted from WT E9-YS and from WT and $L y /-1^{W T / L a c z}$ brain at E10. Lin ${ }^{-} \mathrm{Sca}^{+}{ }^{+} \mathrm{CKit}^{+}$(LSK) progenitors from WT E12 FL were used as positive control. $c M y b$ expression levels, shown on a $\log ^{2}$ scale, were normalized to the mean expression value obtained for WT E10-YS, considered as 1 (Unpaired, two-tailed $t$-Test).

e. Heatmap showing the expression profiles of the DEGs in E9 WT vs E10 WT M $\Phi^{\text {Prim }}$ progenitors that mark the development of tissue resident $\mathrm{M} \Phi$ (Heatmap displays transformed log2-expression values; Unpaired, two-tailed $t$-Test).

f. RT-qPCR analyses of $L y /-1$ expression in $A 1$ to $A 3 M \Phi$ subsets isolated from $\mathrm{Cx} 3 \mathrm{cr} 1^{\mathrm{WT} / \mathrm{GFP}}$ brain at E10. $L y \mid-1$ is expressed by the 3 subsets, with levels decreasing with differentiation. Expression levels were normalized to the mean value obtained for $\mathrm{CX} 3 \mathrm{cr} 1^{\text {WT/GFP }}$ YS A1 progenitors $(n=3)$.

g. Defective differentiation of brain $M \Phi$ progenitor in Lyl-1 mutant embryos. Distribution of A1-A2 and

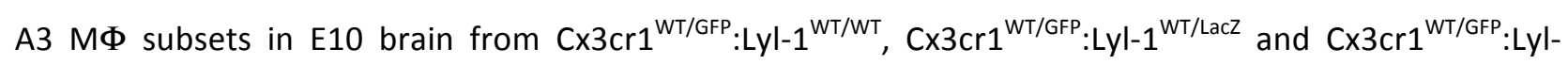
$1^{\text {Lacz/Lacz }}$ embryos. The size of the whole M $\Phi$ population was similar in the three genotypes (Top panel), but Lyl-1 deficiency modified the distribution of the $M \Phi$ subsets (middle and lower panel) with an increased size of the $A 1$ subset and a reduced $A 3$ pool (5-12 independent analyses, each sample cumulating brains from 6-8 embryos. Plots show mean \pm s.e.m.; Unpaired, two-tailed t Test). 


\section{Figure 5: Lyl-1 deficiency leads to transient reductions of the microglia pool at E12 and PO-P3.}

a. Quantification of the microglia population in E12 and E14 brain showing the decreased size of the microglia pool at E12 and its recovery to a normal pool size at E14. Plots show mean \pm s.e.m.; Two tailed, unpaired $t$-test.

b. A reduced microglia proliferation may account for the reduced microglia number at E12, as shown by the two folds decrease (right) of BrdU-labelled cells in Lyl-1 ${ }^{\text {Lacz/Lacz }}$ (middle) compared to WT (left) brains. Plots show mean \pm s.e.m.; Two tailed, unpaired $t$-test.

c. At E12,CX3cr1 ${ }^{\text {WT/GFP }}:$ Lyl-1 ${ }^{\text {LacZ/LacZ }}$ microglia displayed a reduced number and extent of ramifications compared to their $C \times 3 \mathrm{Cr}^{\text {WT/GFP }}$ counterpart. Bottom: Microglia morphology was classified into subtypes depending on the number of main ramifications (A: none, B: 2, C: 3 and D:>3). Top: Microglia deprived of ramifications predominated in $L y /$-1-deficient microglia. 65 and 61 cells were respectively acquired from the midbrain of E12 Cx3cr1 ${ }^{\text {WT/GFP }}$ and $C \times 3 c r 1^{\text {WT/GFP }}:$ Lyl-1 ${ }^{\text {LacZ/LacZ }}$ embryos (for each genotype, brains from 12 embryos were acquired in 3 independent experiments). Microglia were identified by Cx3cr1-driven GFP expression and F4/80-APC immuno-staining. Bar=10mm. Plots show mean \pm s.e.m.; Two tailed, unpaired $t$ test.

d. In $L y \mid-1^{\text {LaczLacz }}$ new-borns, the cellularity of the brain was consistently lower than in WT (left), and so was the estimated microglia number (right). Plots show mean \pm s.e.m.; Two tailed, unpaired $t$-test.

e. Kinetic evolution of $L y l-1$ expression levels in WT microglia from embryonic stages to adulthood. An increased expression of $L y l-1$ from embryonic stages to adulthood was also inferred from timeline RNAseq. data (Matcovitch-Natan et al., 2016) (GEO accession number GSE79812).

f. Quantitative RT-PCR analyses also point to E12 and P0 as key development stages regulated by Lyl-1. $\mathrm{CD} 11 \mathrm{~b}^{+} \mathrm{F} 4 / 80^{+} \mathrm{CD} 45^{\text {low }}$ microglia were isolated at sequential development stages. Bar graphs show the kinetic of expression of genes modified in $L y \mid-1^{\text {Lacz/LacZ }}$ microglia (arrowheads), normalized to the mean expression value in WT E12 microglia $(n=3)$. Error bars indicate s.e.m. Two tailed, unpaired $t$-test.

g. $C \times 3 C r 1$ and $\mathrm{Lyl}-1$ expression in mutant microglia. The expression level of $C \times 3 C R 1$, analysed as in $\mathrm{f}$, was decreased in Lyl-1 mutant at E12 (left), while Lyl-1 expression level was unmodified in CX3CR1 ${ }^{\text {GFP/GFP }}$ microglia at E12 and in new-borns (right).

h. Mafb expression in mutant microglia. Mafb expression level, analysed as in $f$, was reduced in the microglia of $L y /$ - $1^{\text {Lacz/Lacz }}$ new-borns.

i. The expression of genes enriched in microglia and/or essential for their function are deregulated in Lyl-

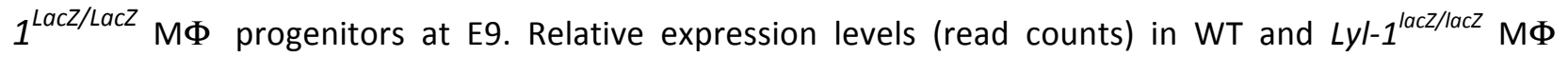
progenitors from E9 YS ( $P$ values were determined by unpaired, two-tailed $t$-Test). 


\section{References}

Bertrand, J.Y., Jalil, A., Klaine, M., Jung, S., Cumano, A., and Godin, I. (2005). Three pathways to mature macrophages in the early mouse yolk sac. Blood 106, 3004-3011.

Mass, E., Ballesteros, I., Farlik, M., Halbritter, F., Gunther, P., Crozet, L., Jacome-Galarza, C.E., Handler, K., Klughammer, J., Kobayashi, Y., et al. (2016). Specification of tissue-resident macrophages during organogenesis. Science.

Matcovitch-Natan, O., Winter, D.R., Giladi, A., Vargas Aguilar, S., Spinrad, A., Sarrazin, S., Ben-Yehuda, H., David, E., Zelada González, F., Perrin, P., et al. (2016). Microglia development follows a stepwise program to regulate brain homeostasis. Science 353 , aad8670. 
Figure 1

a
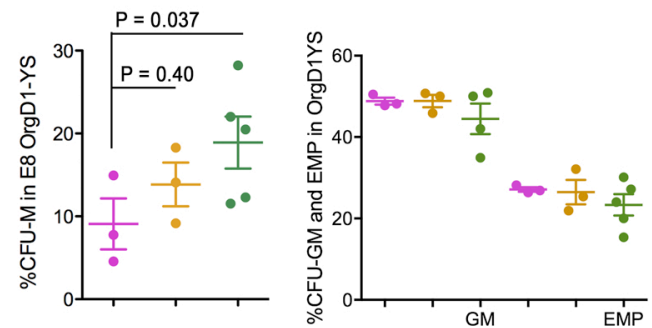

C

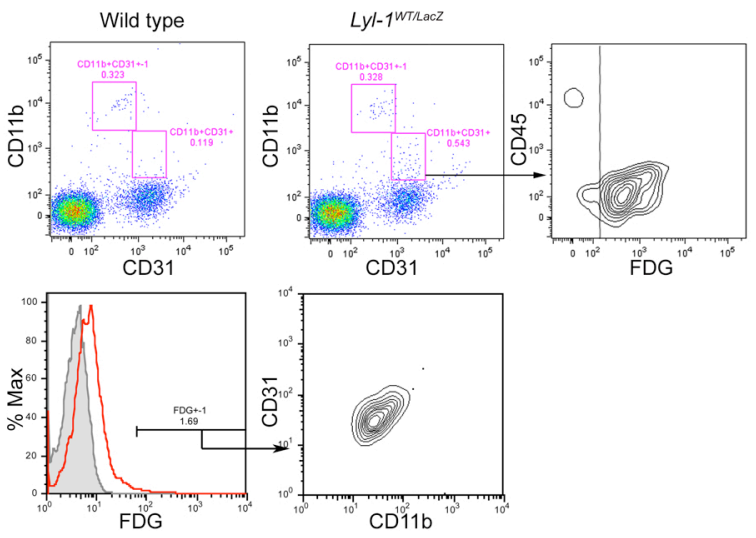

f
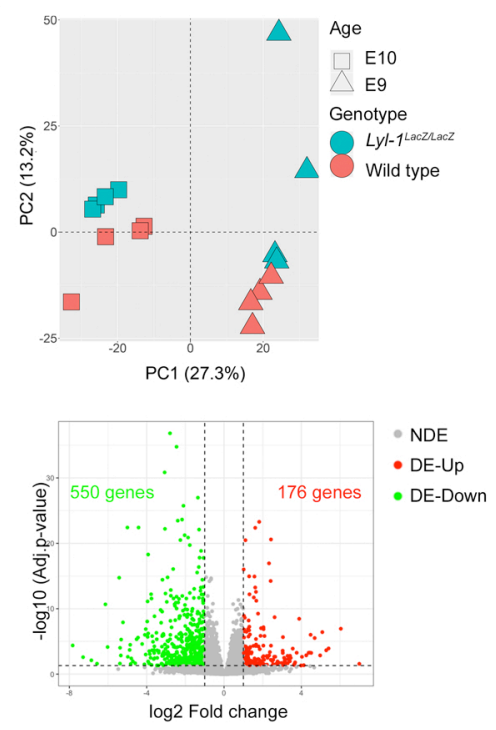

log2 Fold change b

$\mathrm{CKit}^{+} \mathrm{CD} 45^{+} \mathrm{CD} 11 \mathrm{~b}^{+}$gate
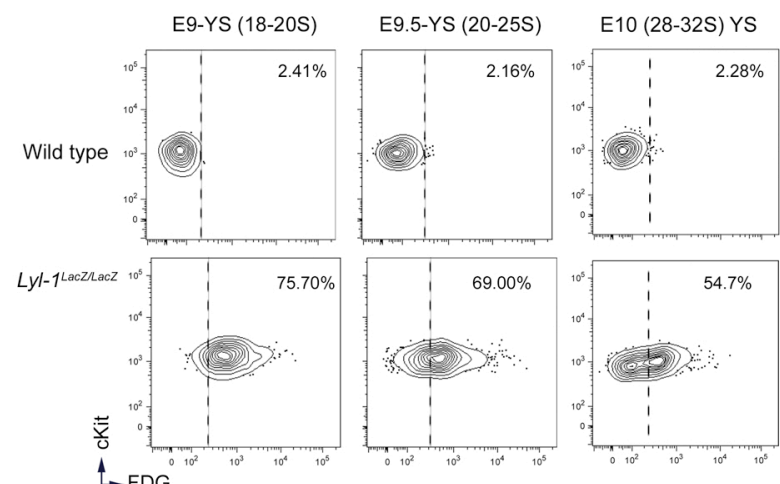

d

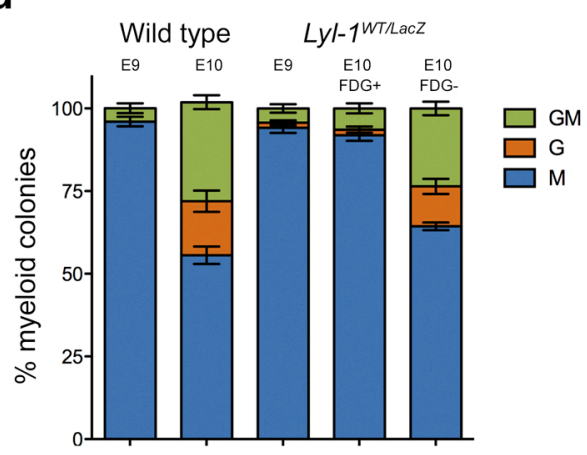

CD11b+ F4/80 gate E10 (28-32S) YS MФ

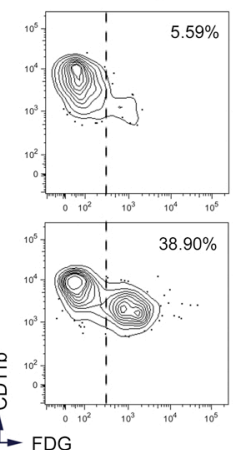

e

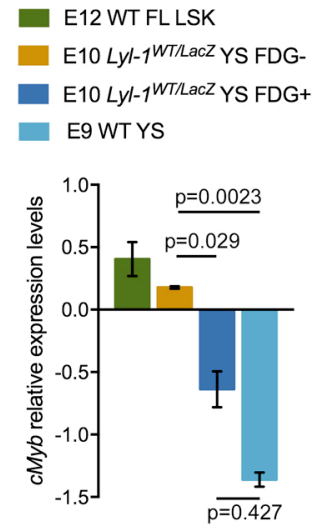

g
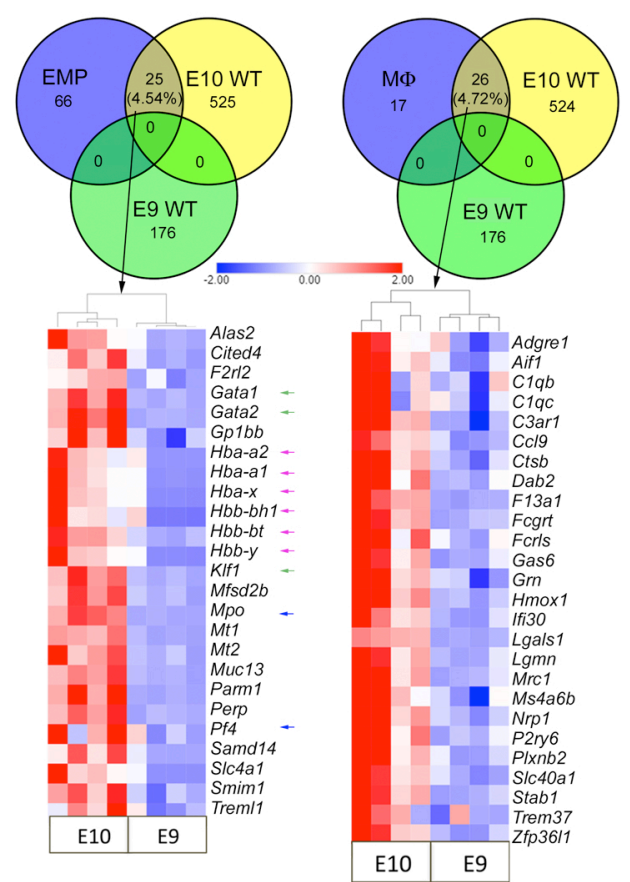

h

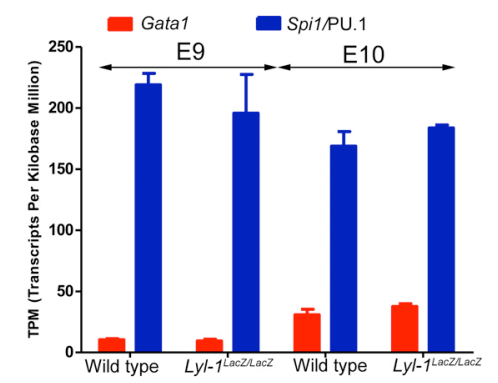

j

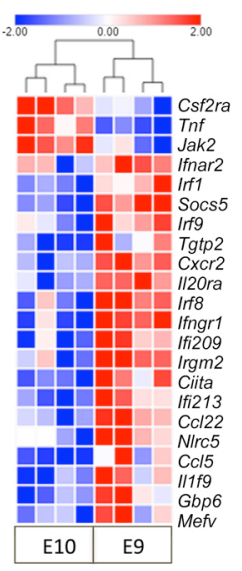

k

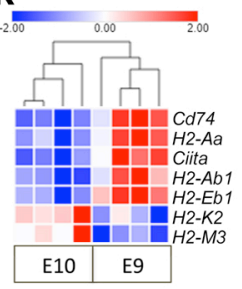

I

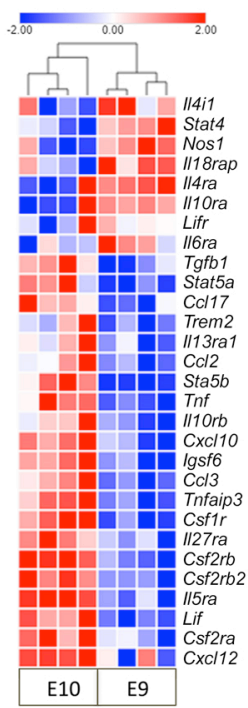

E9 WT vs E10 WT

Role of Pattern Recognition Receptors in Recognition of Bacteria an viruses Inflammasome Pathway

TNFR1 Signaling Colorectal Cancer
Metastasis Signaling ILK Signaling Agrin Interactions at TGF- $\beta$ Signaling

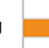
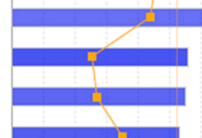
Figure 2

a

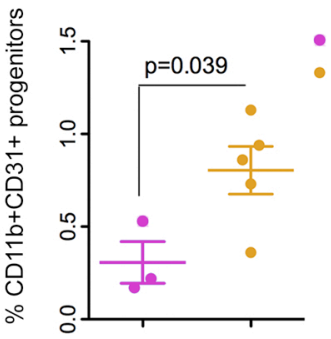

C

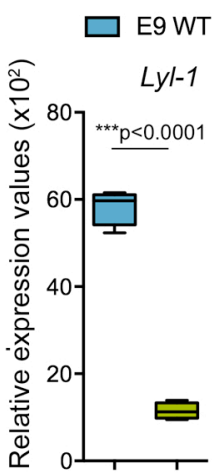

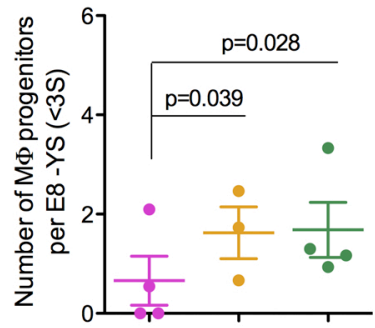

- Wild type

- Lyl-1WT/Lacz

- Lyl-1LacZlacz

\section{b}

E9 Lyl-1LacZ/LacZ

Itga2b/CD41

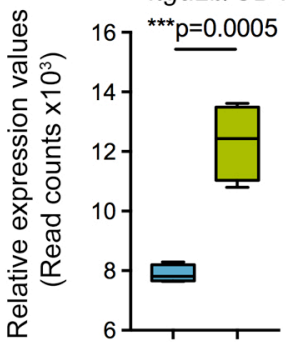

d

E9 Lyl-1 ${ }^{\text {LacZ/LacZ }}$ vs E9 WT

Hypothetical network for drug addiction Ionotropic glutamate receptor pathway - Steroids metabolism Cadherin signaling pathway Cytoplasmic ribosomal proteins $\mathrm{G}$ protein coupled receptor signaling Axon guidance mediated by slit-robo - Butanoate metabolism - Fatty acid biosynthesis $5 \mathrm{HT} 3$ type receptor mediated signaling pathway Adrenaline and noradrenaline biosynthesis Cytoskeletal regulation by rho gtpase Wnt signaling pathway

Nicotinic acetylcholine receptor signaling pathway Neural crest differentiation Metabotropic glutamate receptor group iii pathway G protein coupled receptor signaling-G alpha Epac and Erk - Fructose mannose metabolism $G$ protein coupled receptor signaling- $G$ alpha $G$ protein coupled receptor signaling-G Pertusis toxin

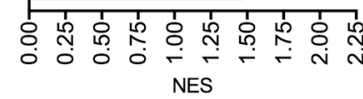

Regulation of synaptic transmission
Presynaptic membrane
Embryonic skeletal system development
Regulation of membrane potential
Postsynaptic membrane
Excitatory synapse
Embryonic digit morphogenesis
Anterior posterior pattern specification
Cell junction
Collagen
of long-term neuronal synaptic plasticity
Terminal button
Extracellular matrix structural constituent
Inner ear morphogenesis
Response to cocaine
Bone morphogenesis
MPA selective glutamate receptor activity
involved in regulation of gene expression

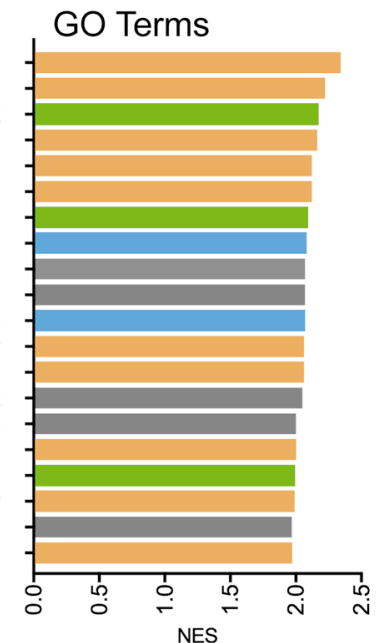




\section{Figure 3}

a

\section{E10 Yolk sac}

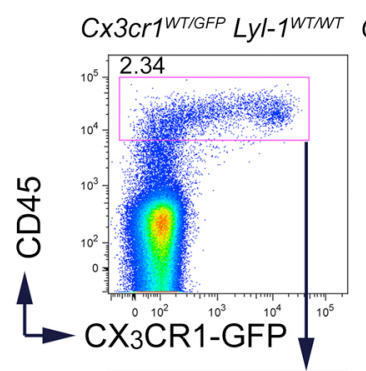

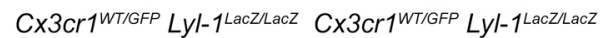
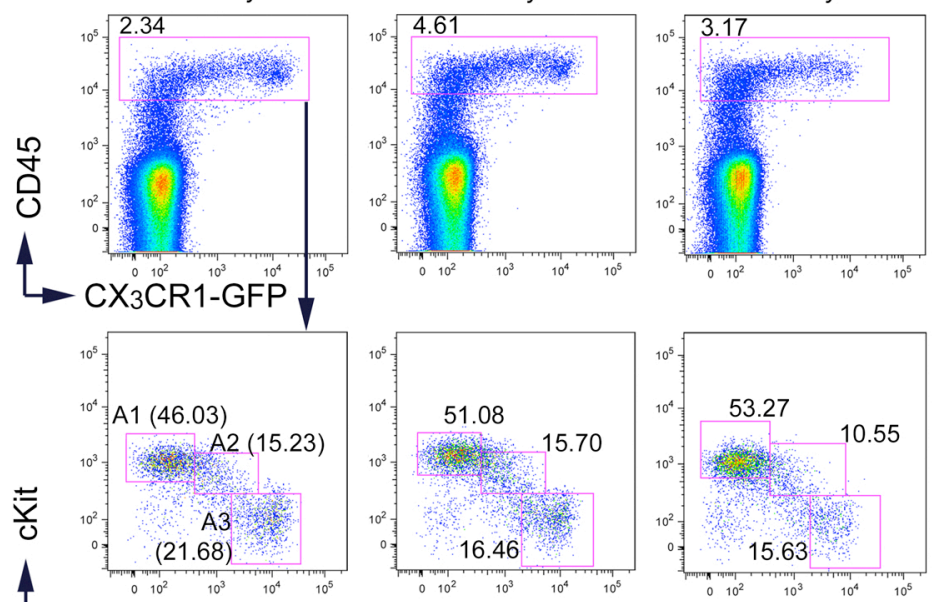

CX 3 CR1-GFP
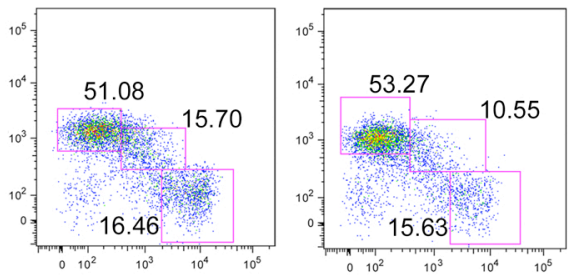

- Cx3cr1WT/GFP Lyl-1WTMT

- Cx3cr1WT/GFP Lyl-1WT/Lacz

- Cx3cr1WT/GFP Lyl-1LacZ/Lacz
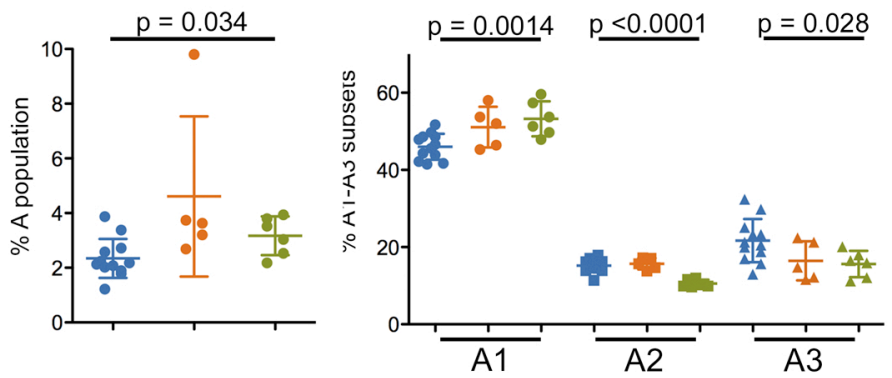

b

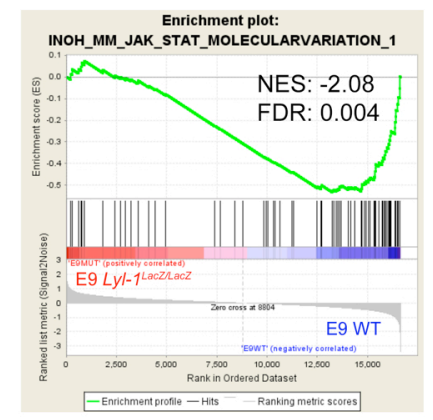

C

$\square$ E9 WT $\square$ E9 Lyl-1 LacZlLacZ

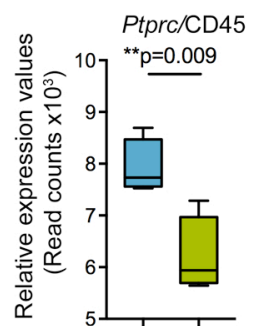

Csfr1

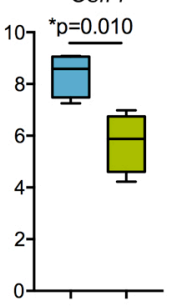

Itgam/CD11b

Cd33
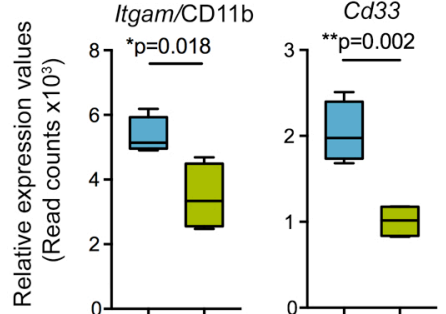

d
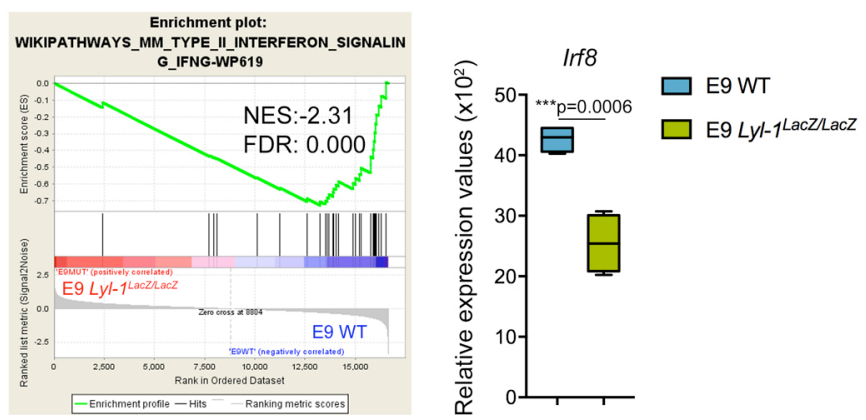

e

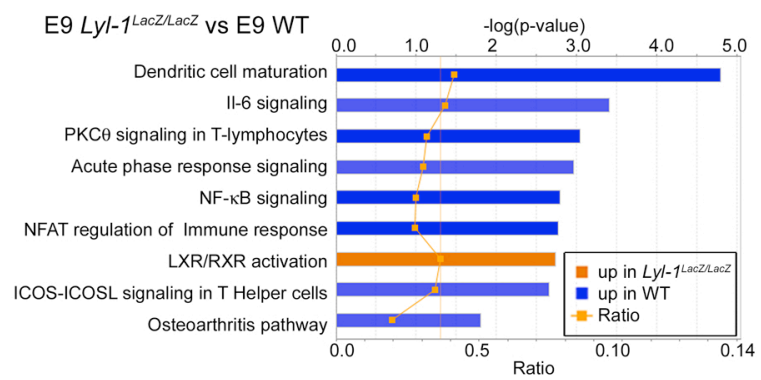

f

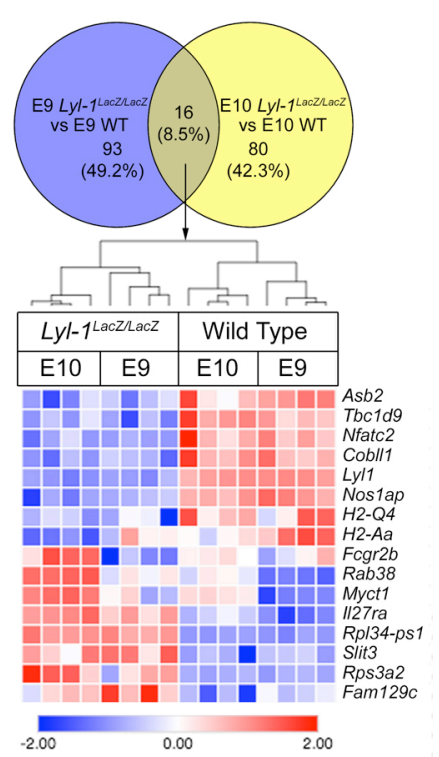


Figure 4

a

$\mathrm{cKit}^{+} \mathrm{CD} 45^{+} \mathrm{CD} 11 \mathrm{~b}^{+}$gate

E10 (28-32S) Brain E10 Foetal liver
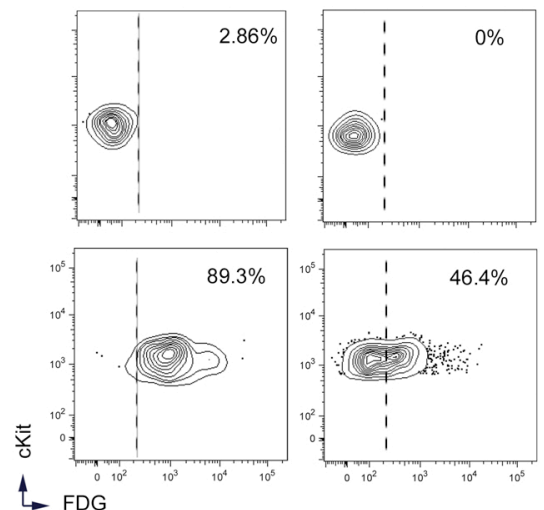

C
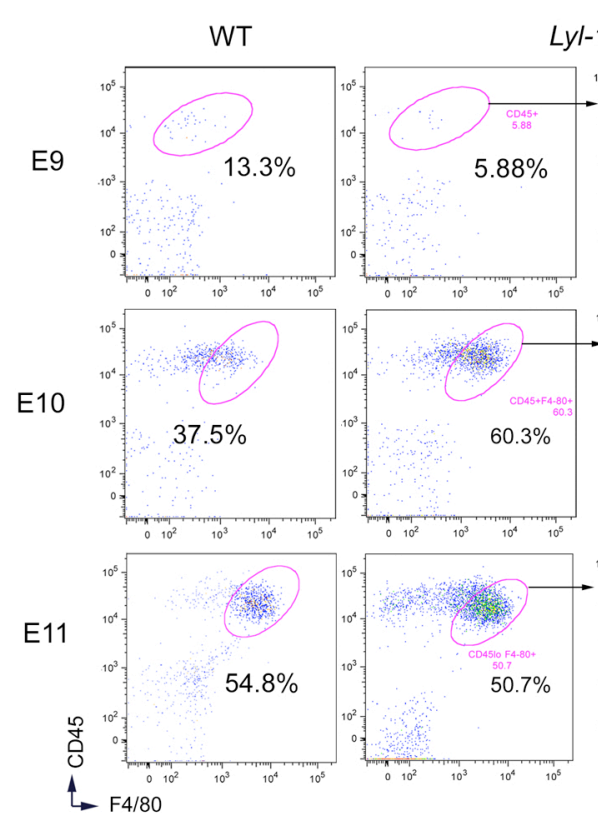

Lyl-1WTLacz
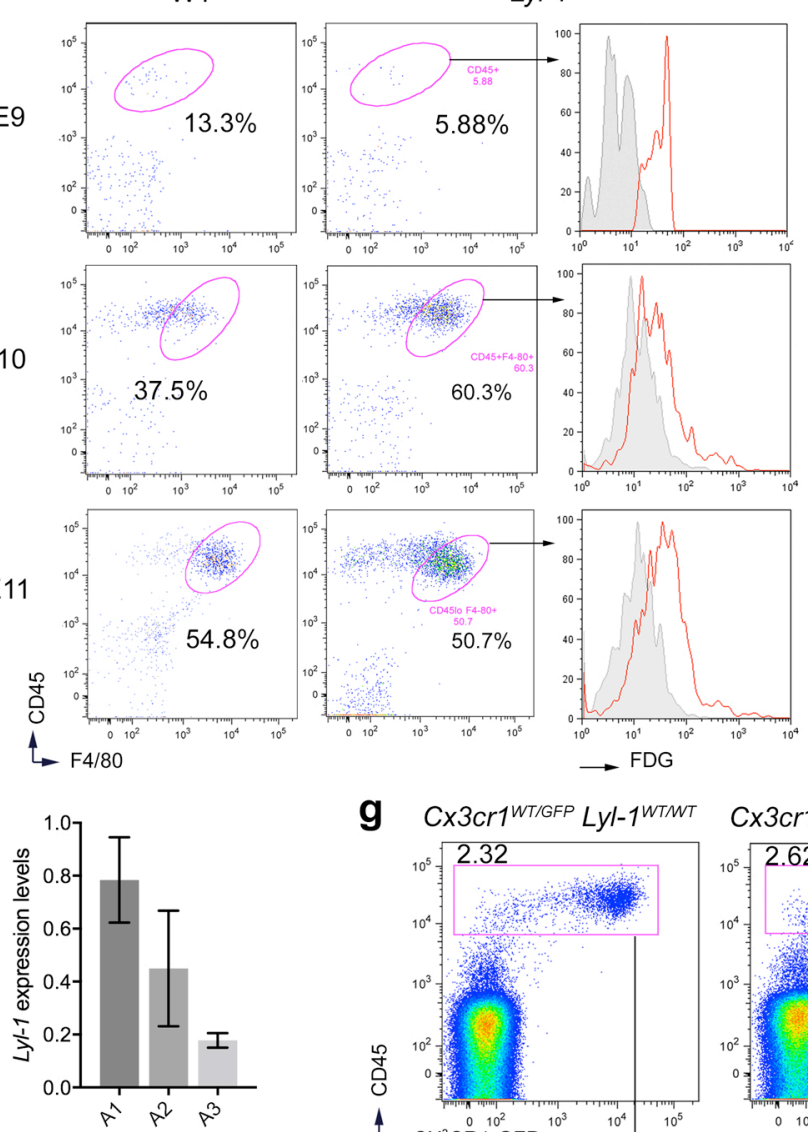

g $C \times 3 C r 1^{W T / G F P} L y l-1^{W T N T}$
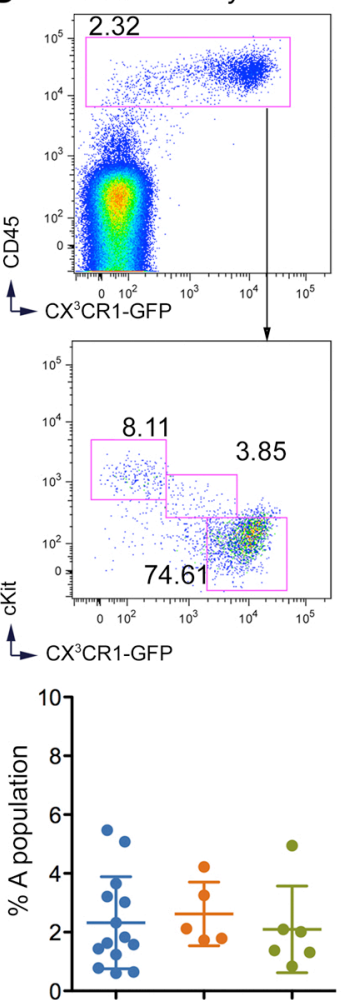

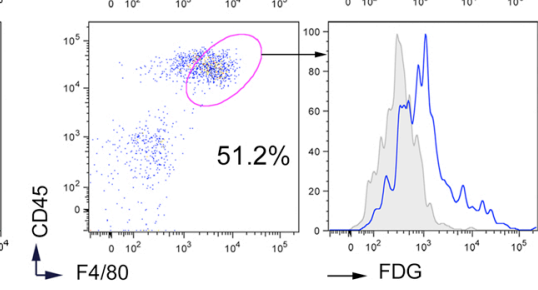

$\mathrm{CD} 11 \mathrm{~b}+\mathrm{F} 4 / 80+$ gate E10 (28-32S) Brain
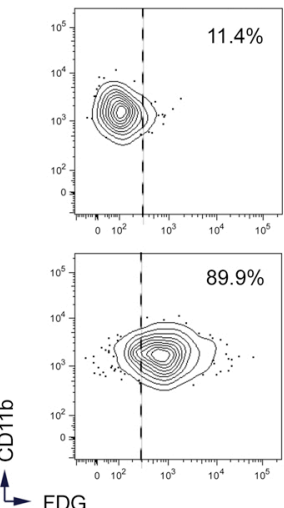

$89.9 \%$
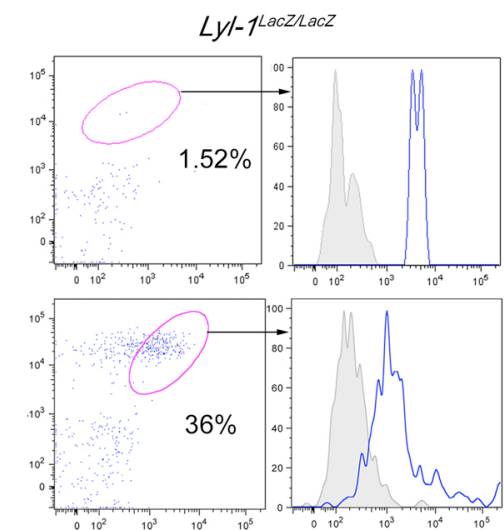

Cx3cr1WT/GFP $L y l-1^{\text {LacZLLaCZ }}$ Cx3cr1WT/GFP Lyl-1 LacZLacZ
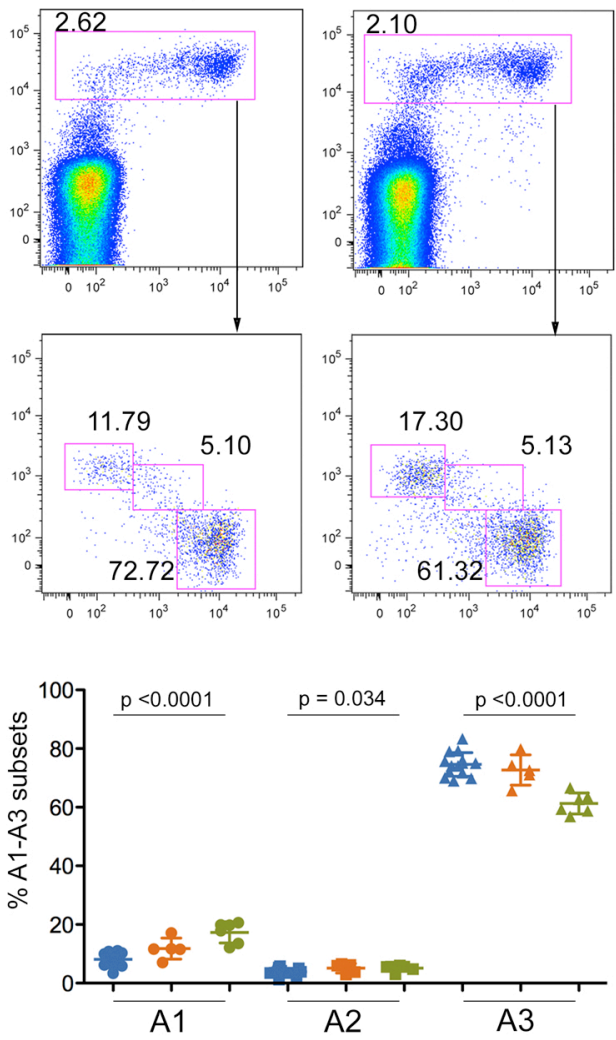

d

E12 WT FL LSK

E9 WT YS

E10 Lyl-1WT/LacZ Brain E10 WT Brain

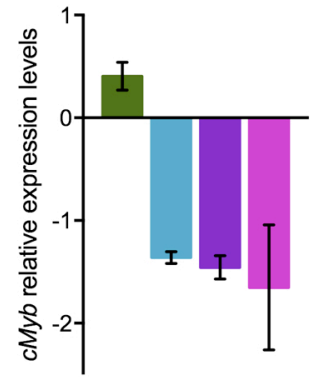

e

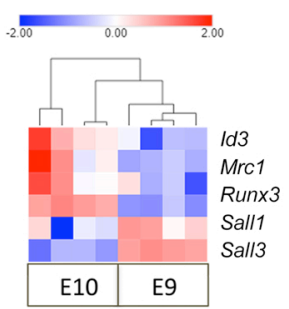

- Cx3cr1WT/GFP Lyl-1WTNT

- Cx3cr1WT/GFP $L y l-1$ WT/Lacz

- Cx3cr1WT/GFP $L y l-1^{\text {LacZLacZ }}$ 
Figure 5

a

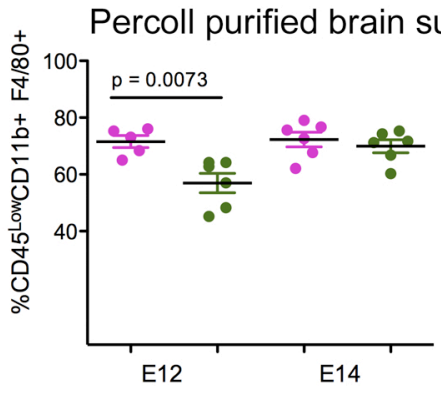

C

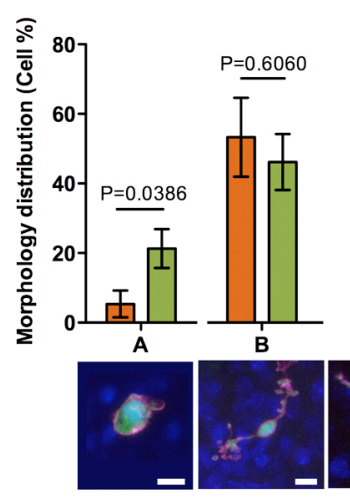

f

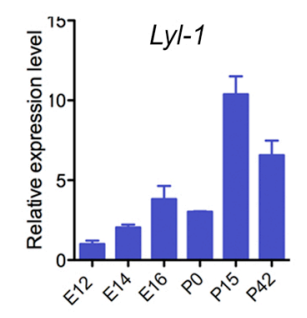

g

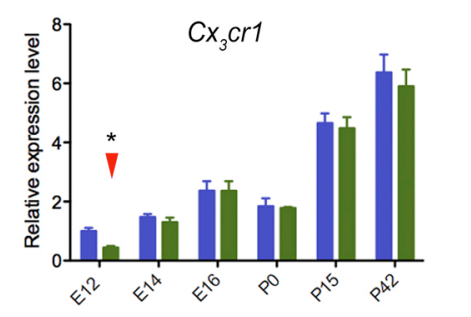

b

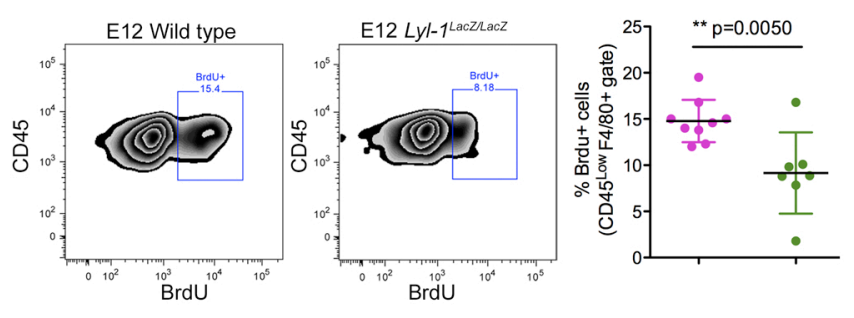

d spension

- Wild type

- Lyl-1'Laczlacz $\square C \times 3 c r 1^{W T / G F P}$

$\square$ Cx3cr1 ${ }^{\text {WT/GFP }}$ Lyl-1 1LacZLacZ
- P0-P3 Wild type

- P0-P3 Lyl-1 1 Laczracz
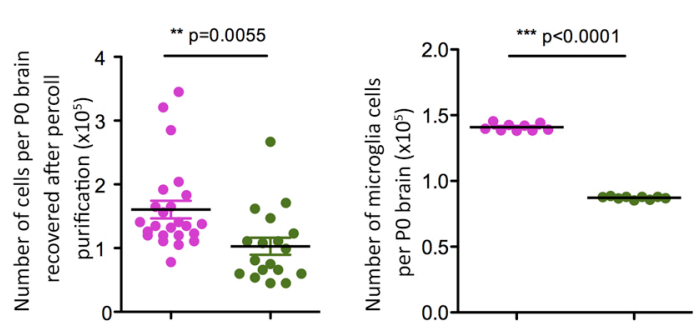

$\mathrm{CX}_{3} \mathrm{CR} 1 \mathrm{~F} 4 / 80 \mathrm{DAPI}$
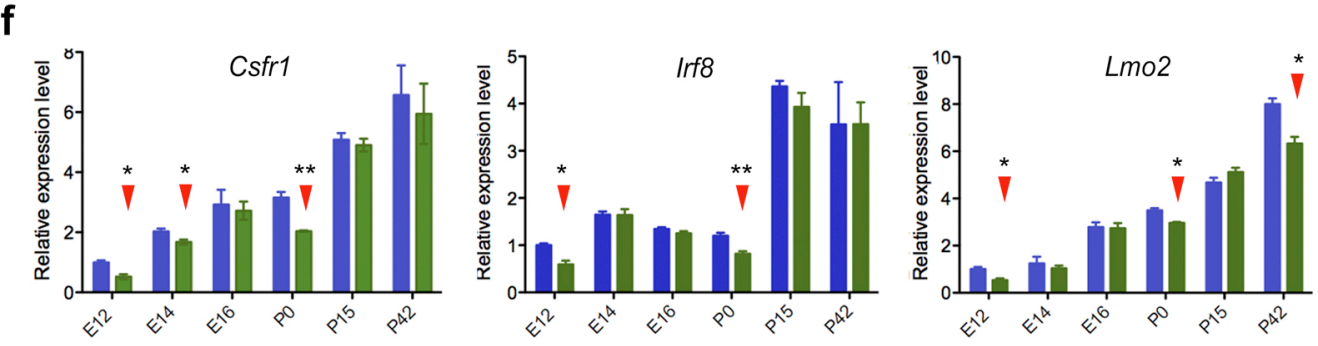

h
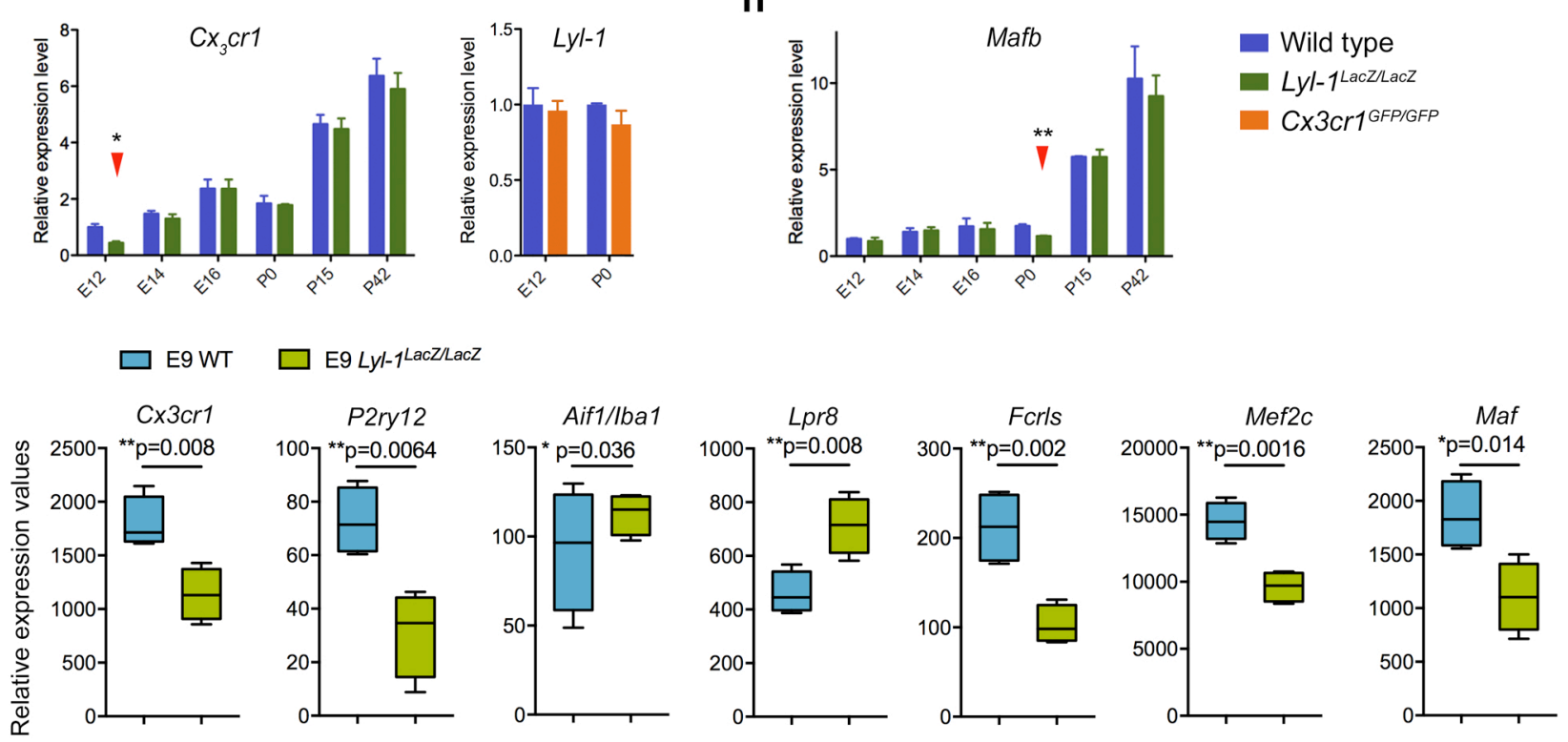